\title{
Dynamic Analysis of Self-Oscillating H-Bridge Inverters with State Feedback
}

\author{
Luis Benadero ${ }^{1}$, Francisco Torres ${ }^{2}$, Enrique Ponce ${ }^{2}$, Abdelali El Aroudi ${ }^{3}$
}

\begin{abstract}
This paper presents a comprehensive approach to analyze the dynamics of a generalized model of resonant inverters using nonsmooth dynamical system theory. The model simultaneously covers both parallel and series resonant inverters under state feedback control. The multi-parametric physical space is reduced to a plane, which is divided in several regions with different dynamical behavior. The boundaries separating these regions are located by solving their corresponding equations and it is found that they all emerge from a singular point in the parameter plane. Suitability for applications of these regions is emphasized, thus providing useful criteria for parameter selection.
\end{abstract}

Keywords: Resonant inverters, State feedback control, Piecewise linear dynamics, Sliding dynamics, Bifurcations, Limit cycles

\section{Introduction}

Switched-mode power converters are widely used for power sources and power management systems in different industrial applications such as portable devices, efficient solid-state lighting drivers and technologies for renewable energy production among others [1]. Nevertheless, despite their widespread use, one of their main drawbacks is their switching nonlinearity making them prone to exhibit a variety of complex dynamics. Resonant converters have been studied since the 80's [2,3]. However, these specific converters have not been widely used in industrial applications due to their complex dynamics and the required sophisticated tools for their analysis and design. Previous studies have mainly focused on approximate approaches to deal with their dynamic and steady-state characteristics. Recently, resonant converter topologies have gained renewed attention from the power electronics community, so that they are emerging in different consumer electronics applications such as electrical vehicle battery chargers, efficient LED drivers, servers and telecommunications [4]. This is mainly due to their potential to achieve high efficiency and high power density at the same time $[4,5]$. The use of resonant converters in many emerging applications may not only be advantageous but also necessary. Therefore, it is indispensable to deeply understand their behavior using accurate approaches.

The study of the dynamic behavior of these systems has attracted and is still attracting the interest of many researchers $[6,7,8,9,10]$. The approaches used can be broadly classified as either time-domain or frequency-domain

${ }^{3}$ Universitat Rovira i Virgili (URV), Departament d'Enginyeria Electrònica, Elèctrica i Automàtica (DEEEA), Tarragona, Spain
} 
techniques. Time-domain methods are based either on the solution of time-domain differential equations in the state plane [2] or on the time-domain Hamel locus [11]. Frequency-domain methods are based on harmonic balance methods which is a general approach for analyzing the existence and stability of limit cycles in nonlinear systems in the in the context of nonlinear control theory. Describing function (DF) also called First Harmonic Approximation (FHA) method in the context of resonant inverters is an approximate frequency-domain technique derived from the general Harmonic Balance (HB) approach. It has been widely applied to characterize the limit cycle behavior of resonant converters $[9,10,12,13]$. In the FHA approach, it is assumed that the quality factor is sufficiently high such that the switching frequency is very close to the resonant frequency and that the waveforms of the state variables have very low harmonic distortion [7, 8]. While the FHA approach gives enough accurate results for high values of the quality factor because of the weak nonlinearity taking place, it is no longer valid in the opposite case and significant errors appear making them to fail short in predicting the actual behavior of the system [14], since strong nonlinearity effects appear for low values of quality factors. Hence, the FHA approach is an approximate technique and cannot provide accurate prediction of the system behavior for all loading conditions, as shown in Section 7.1. On the contrary, the time-domain state-plane approach allows one to accurately uncover the dynamical behavior without any approximation [2]. Another novel accurate approach is based on the locus of a perturbed relay system (LPRS) frequency-domain technique $[15,16]$. This method, which is briefly exposed in Section 7.2 , accurately predicts the converter amplitude and frequency and was successfully applied to a buck converter under sliding mode control [16]. Discrete-time modeling is another accurate tool, which were used in $[17,18]$, to determine the possible steady-state operating points of different structures of resonant converters. A drawback of discrete-time approaches is that the switching pattern must be known before hand and the possibility for sliding-motion to take place is ignored. This makes them to fail short in revealing the real global dynamics of the system. Sliding motion can take place in this kind of systems $[19,20]$. In particular, a trajectory of the switched system can partly remain on a sliding-mode region associated with an infinite number of switching between two different subsystems and a part of a limit cycle may emerge on this region. Such dynamical behavior results in nonsmooth complex behaviors that cannot be described by the previous conventionally used approaches.

In this paper, the non-smooth model of a generalized resonant inverter with state feedback is considered. The model covers both parallel and series resonant inverters under state feedback control. While in [22] the inverter was operating under a Zero Current Switching (ZCS) control, by using only the inductor current in the switching decision, here the control is generalized, so that the capacitor voltage also intervenes in the feedback. As a consequence, the structural parameter $\beta$, defined in [22] and conveniently redefined here, is no more limited to nonnegative values, thus enriching the variety of possible dynamics. Actually, we will see that the oscillatory behavior is facilitated by the inclusion of the voltage feedback, even in the case of a quality factor of the circuit $(Q)$ below the critical value $1 / 2$.

A comprehensive approach to analyze and design the inverter using non-smooth dynamical theory is presented. The relevance of computing the different bifurcations is stressed, since only thanks to the knowledge of the complete bifurcation set it is possible to get a global overview of the qualitatively different dynamical regimes. In particular, an essential parameter plane is characterized, where several bifurcation curves appear, organized around a high codimension bifurcation point. These curves define the parameter regions with similar qualitative behavior and its computation not only determines the 'safe' region of parameter values where the system is robust, but also provides important keys for possible control actions pursuing to enlarge such a safe region. Recently, a complementary analysis of these self-resonant inverters providing some experimental results is in [23] and a variety of proposals to circumvent the multistability drawback are worked out in [24].

The outline of the paper is as follows. First, the system under study is described and mathematically modeled in Section 2. The equilibrium points and the dynamics around the switching manifold are introduced in Section 3 . The main results are summarized in Section 4. In Section 5, existence and properties of booth crossing and sliding limit cycles are studied. Some hints on applications are given in Section 6. In Section 7, the analysis given in this paper is compared to other existing approaches such as DF and the locus of a perturbed relay system (LPRS) techniques, thus showing that although these more general methods are easier to be applied, they can undergo several limitations in order to capture all the different dynamical behaviors that our circuit can exhibit. Finally, conclusions are drawn in the last section. 


\section{Mathematical model}

\subsection{Circuit description}

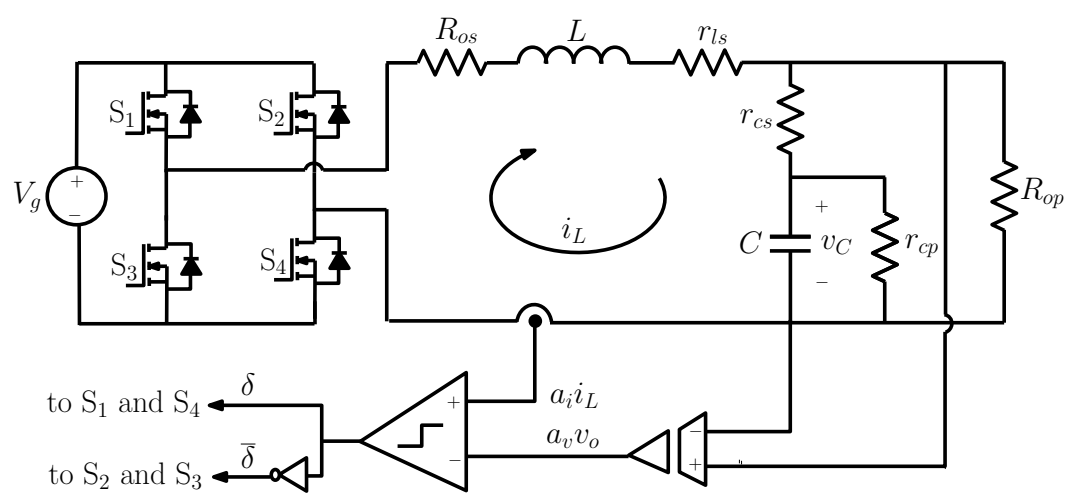

Figure 1. Schematic diagram of the resonant inverter.

Figure 1 shows a generalized circuit diagram representing both parallel and series LC resonant inverter, taking into account their parasitic parameters. There are applications where the system is used for supplying DC loads like for charging a battery. In this case, a nonlinear rectifier is inserted at the output of the inverter. In that cases, much of the existing works use an approximate analysis by considering the nonlinear rectifier together with the resistive load as an equivalent resistance [1]. Here, we are more interested in applications where such circuits are used for supplying AC loads and in this case, the load is connected to the inverter without rectifier, for instance, in induction heating [25].

The control strategy used in [22] is generalized here by considering both inductor current and output voltage in the feedback. In the diagram, there can be identified the output series resistance $R_{o s}$; the inductor with inductance $L$ and parasitic series resistor $r_{l s}$; the output parallel conductance $G_{o p}=1 / R_{o p}$; the capacitor with capacitance $C$, parasitic parallel conductance $g_{c p}=1 / r_{c p}$ and parasitic series resistance $r_{c s}$. Note that with the above notation, for the series topology, the parallel resistor is replaced by an open circuit and then $G_{o p}=0$. Likewise, for the parallel topology, the series resistor is replaced by a short circuit and so $R_{o s}=0$. Other elements in the circuit diagram in Fig. 1 are the input source voltage $V_{g}$, and the switches $\mathrm{S}_{1}-\mathrm{S}_{4}$. Let $v_{C}$ be the voltage of the capacitor $(C), i_{L}$ be the current through the inductor $(L)$ and $v_{o}$ be the voltage drop across the output resistance $\left(R_{o p}\right)$. Besides, $a_{i}$ and $a_{v}$ are the gain parameters used in the state feedback control, related to the inductor current $i_{L}$ and the output voltage $v_{o}$ sensors.

The circuit operation is based on an automatically activated switching between two configurations driven by the binary signal $\delta \in\{0,1\}$ under the following control rules: switches $\mathrm{S}_{1}$ and $\mathrm{S}_{4}$ are on when $a_{i} i_{L}>a_{v} v_{o}$ (therefore $\delta=1)$, and they are turned off when $a_{i} i_{L}<a_{v} v_{o}(\delta=0)$. Switches $\mathrm{S}_{2}$ and $\mathrm{S}_{3}$ are driven in a complementary way to $S_{1}$ and $S_{4}$. It is clear that if there is no switching, the capacitor voltage and the inductor current tend to constant values. In other words, there will be an equilibrium point with positive values if $\delta=1$, and its symmetrical point with negative values if $\delta=0$. Consequently, depending on the starting conditions, the dynamics could be addressed to one of these undesired equilibrium.

Taking into account that $i_{L}=i_{c s}+G_{o p} v_{o}$, in which $i_{c s}=\left(v_{o}-v_{C}\right) / r_{c s}$ is the current trough the resistance $r_{c s}$, then the voltage drop across the output parallel resistance $R_{o p}$ is given by $v_{o}=\kappa\left(v_{C}+r_{c s} i_{L}\right)$, in which

$$
\kappa=\frac{1}{1+r_{c s} G_{o p}} .
$$

The switching condition can therefore be expressed in terms of the state variables, $v_{C}$ and $i_{L}$, as follows

$$
a_{i} i_{L}-a_{v} v_{o}=\left(a_{i}-\kappa a_{v} r_{c s}\right) i_{L}-\kappa a_{v} v_{C}=\left(a_{i}-\kappa a_{v} r_{c s}\right)\left(i_{L}-g_{C} v_{C}\right)=0,
$$

where $g_{C}$ is an effective control factor, with conductance dimension, defined as

$$
g_{C}=\frac{a_{v} \kappa}{a_{i}-\kappa a_{v} r_{c s}} .
$$


Since $0<\kappa \leq 1$ and $r_{c s}$ is small in practice, it turns out that $\kappa$ is close to 1 and the factor $g_{C}$ differs only slightly from the ratio $a_{v} / a_{i}$. Moreover, since the value $a_{i}$ must be selected positive in practical applications, the analysis will be restricted to the case $a_{i}-\kappa a_{v} r_{c S}>0$. Note that the variable $u=2 \delta-1$ is determined by a control law such that $u=1$ (that is $\delta=1$ ) if $i_{L}>g_{C} v_{C}$, and $u=-1$ (that is $\delta=0$ ) if $i_{L}<g_{C} v_{C}$. Then, the switching condition $h_{s}\left(v_{C}, i_{L}\right)=0$ does not depend explicitly on time and therefore, the system is autonomous, being the function $h_{s}$ given by

$$
h_{s}\left(v_{C}, i_{L}\right)=i_{L}-g_{C} v_{C} .
$$

By applying Kirchoff laws to the circuit depicted in Fig. 1, the following dynamical model is obtained

$$
\frac{d}{d t}\left(\begin{array}{c}
v_{C} \\
i_{L}
\end{array}\right)=\mathbf{A}_{0}\left(\begin{array}{c}
v_{C} \\
i_{L}
\end{array}\right)+u \mathbf{b}_{0},
$$

where the matrix $\mathbf{A}_{0}$ and the vector $\mathbf{b}_{0}$ are given by

$$
\mathbf{A}_{0}=\left(\begin{array}{cc}
-\frac{G_{P}}{C} & \frac{\kappa}{C} \\
-\frac{\kappa}{L} & -\frac{R_{S}}{L}
\end{array}\right), \quad \mathbf{b}_{0}=\left(\begin{array}{c}
0 \\
\frac{V_{g}}{L}
\end{array}\right),
$$

and the equivalent series resistance $R_{S}$ and parallel conductance $G_{P}$ are defined as

$$
R_{S}=R_{o s}+r_{l s}+\kappa r_{c s}, G_{P}=g_{c p}+\kappa G_{o p}
$$

Hence, the natural frequency $\omega_{0}$ and the quality factor $Q$ are as follows

$$
\begin{aligned}
& \omega_{0}=\sqrt{D_{0}}=\sqrt{\frac{R_{S} G_{P}+\kappa^{2}}{L C},} \\
& \frac{1}{Q}=-\frac{T_{0}}{\omega_{0}}=\frac{G_{P}}{\omega_{0} C}+\frac{R_{S}}{\omega_{0} L},
\end{aligned}
$$

where $D_{0}$ and $T_{0}$ stand for the determinant and the trace of matrix $\mathbf{A}_{0}$ respectively.

Next, in order to simplify the analysis of its possible dynamics and bifurcations, system (2)-(3) will be normalized into a simpler form with a minimum number of parameters.

\subsection{Canonical forms}

Let us first redefine the state variables in the form

$$
\tilde{v}=v_{C}, \quad \tilde{i}=i_{L}-g_{C} v_{C},
$$

such that the condition $\tilde{i}=0$ defines the switching manifold. Then, system (2)-(3) reduces to

$$
\begin{aligned}
\frac{d}{d t}\left(\begin{array}{c}
\tilde{v} \\
\tilde{i}
\end{array}\right) & =\tilde{\mathbf{A}}_{0}\left(\begin{array}{c}
\tilde{v} \\
\tilde{i}
\end{array}\right)+u \tilde{\mathbf{b}}_{0}, \\
h(\tilde{v}, \tilde{i}) & =\tilde{i}
\end{aligned}
$$

in which

$$
\tilde{\mathbf{A}}_{0}=\left(\begin{array}{cc}
\frac{\kappa g_{C}-G_{P}}{C} & \frac{\kappa}{C} \\
-\frac{\kappa}{L}-g_{C}\left(\frac{R_{S}}{L}+\frac{\kappa g_{C}-G_{P}}{C}\right) & -\frac{R_{S}}{L}-\frac{\kappa g_{C}}{C}
\end{array}\right), \quad \tilde{\mathbf{b}}_{0}=\mathbf{b}_{0} .
$$

Notice that the natural frequency $\omega_{0}$ and the quality factor $Q$ remain unchanged since matrices $\mathbf{A}_{0}$ and $\tilde{\mathbf{A}}_{0}$ are similar. 
In searching for a canonical form, let $\tau=\omega_{0} t$ and redefine the state vector as follows

$$
\tilde{\mathbf{x}}=\left(\begin{array}{c}
\tilde{x}_{1} \\
\tilde{x}_{2}
\end{array}\right)=\left(\begin{array}{cc}
\frac{C\left(\kappa+g_{C} R_{S}\right)+g_{C} L\left(\kappa g_{C}-G_{P}\right)}{C V_{g}} & \frac{L\left(\kappa g_{C}-G_{P}\right)}{C V_{g}} \\
0 & \frac{\omega_{0} L}{V_{g}}
\end{array}\right)\left(\begin{array}{c}
\tilde{v} \\
\tilde{i}
\end{array}\right) .
$$

Let also define the new parameter

$$
\beta=\frac{\left(G_{P}-\kappa g_{C}\right) L}{L G_{P}+C R_{S}}
$$

which can be varied by means of the control factor $g_{C}$. Note that the value of $\beta$ can be selected in a wide real range. Therefore, the following result is obtained.

Proposition 1. System (2)- (3) can be reduced to the form

$$
\begin{aligned}
\frac{d \tilde{\mathbf{x}}}{d \tau} & =\tilde{\mathbf{A}} \tilde{\mathbf{x}}+u \tilde{\mathbf{b}}, \\
h(\tilde{\mathbf{x}}) & =\tilde{x}_{2},
\end{aligned}
$$

where matrix $\tilde{\mathbf{A}}$ and vector $\tilde{\mathbf{b}}$ are

$$
\tilde{\mathbf{A}}=\left(\begin{array}{cc}
0 & 1 \\
-1 & \frac{-1}{Q}
\end{array}\right), \quad \tilde{\mathbf{b}}=\left(\begin{array}{c}
-\frac{\beta}{Q} \\
1
\end{array}\right),
$$

and $u=1$ if $h(\tilde{\mathbf{x}})>0$ and $u=-1$ if $h(\tilde{\mathbf{x}})<0$, or equivalently $u=\operatorname{sign}(h(\tilde{\mathbf{x}}))$.

Remark 1. Notice that if the parasitic elements can be neglected so that $\kappa=1, g_{C}=a_{v} / a_{i}, R_{S}=R_{o s}, G_{P}=G_{o p}$, then

$$
\omega_{0}=\sqrt{\frac{R_{o s} G_{o p}+1}{L C}}, \quad \frac{1}{Q}=\frac{G_{o p}}{\omega_{0} C}+\frac{R_{o s}}{\omega_{0} L}, \quad \beta=\frac{\left(G_{o p}-a_{v} / a_{i}\right) L}{L G_{o p}+C R_{o s}} .
$$

For each one of the two linear subsystems in (7), the natural frequency is the unity and the quality factor is $Q$. Let $p^{ \pm}$be the eigenvalues of matrix $\tilde{\mathbf{A}}$, i.e.,

$$
p^{ \pm}=-\frac{1}{2 Q} \pm \sqrt{\frac{1}{4 Q^{2}}-1} .
$$

Different cases appear depending on the quality factor $Q$. If $Q>1 / 2$ there is a pair of complex conjugate eigenvalues, if $Q=1 / 2$ there is one double real eigenvalue and when $0<Q<1 / 2$ there are two real eigenvalues.

Considering these cases, system (7)-(8) can be reformulated by means of an auxiliary modal variable, as in [21], in order to get easier expressions when studying the bifurcation set of our circuit. For that, let define first the [tri]modal parameter $m \in\{j, 0,1\}$, where $j$ is the imaginary unit, in order to account for the three cases stated above, that is

$$
m=j \text { when } Q>1 / 2, \quad m=0 \text { when } Q=1 / 2, \quad m=1 \text { when } 0<Q<1 / 2 .
$$

For convenience, the parameters $\gamma$ and $v$ depending on the parameter $Q$ are defined as follows,

$$
\gamma=\left\{\begin{array}{cl}
-1, & \text { if } Q=1 / 2, \\
\frac{-1}{\sqrt{\left(1-4 Q^{2}\right) m^{2}}}, & \text { if } Q \neq 1 / 2
\end{array} \quad v=\frac{-1}{2 Q \gamma} .\right.
$$

In Fig. 2, $\gamma(Q)$ is represented for $m=1$, i.e., $Q<1 / 2$ and for $m=j$, i.e., $Q>1 / 2$. Note that $\gamma<0$ in the three modes.

Then, if a new state vector $\mathbf{x}=\left(x_{1}, x_{2}\right)$ and a new time $\theta$ are defined as

$$
x_{1}=\tilde{x}_{1}, \quad x_{2}=v \tilde{x}_{2}, \quad \theta=v \tau,
$$

the following proposition results. 


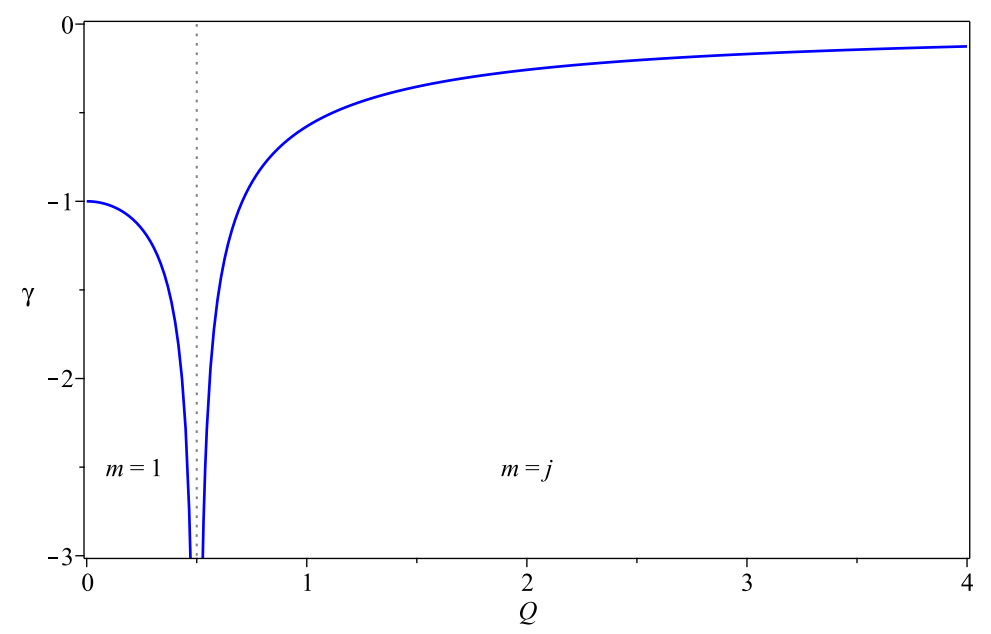

Figure 2. Plot of function $\gamma$ versus $Q$ for modes $m=1$ (i.e., $Q<1 / 2$ ) and $m=j$ (i.e., $Q>1 / 2$ ).

Proposition 2. System (7)-(8) can be reduced to

$$
\begin{aligned}
\frac{d \mathbf{x}}{d \theta} & =\mathbf{A x}+u \mathbf{b}, \\
h(\mathbf{x}) & =x_{2},
\end{aligned}
$$

where matrix $\mathbf{A}$ and vector $\mathbf{b}$ are

$$
\mathbf{A}=\left(\begin{array}{cc}
0 & \gamma^{2}-m^{2} \\
-1 & 2 \gamma
\end{array}\right), \quad \mathbf{b}=\left(\begin{array}{c}
2 \beta \gamma \\
1
\end{array}\right)
$$

and $u=\operatorname{sign}(h(\mathbf{x}))$.

According to (11), the switching manifold $\Sigma$ is defined as

$$
\Sigma=\left\{\mathbf{x}=\left(x_{1}, x_{2}\right): x_{2}=0\right\}
$$

and so the state space of the canonical form has two linearity regions, namely

$$
\Sigma^{+}=\left\{\mathbf{x}=\left(x_{1}, x_{2}\right): x_{2}>0\right\}, \quad \Sigma^{-}=\left\{\mathbf{x}=\left(x_{1}, x_{2}\right): x_{2}<0\right\} .
$$

In accordance to the defined switching manifold $\Sigma$ and the two corresponding regions $\Sigma^{+}$and $\Sigma^{-}$, system (10)-(11) can be rewritten as

$$
\frac{d \mathbf{x}}{d \theta}=\mathbf{F}(\mathbf{x})=\left\{\begin{array}{l}
\mathbf{F}^{+}(\mathbf{x})=\left(F_{1}^{+}(\mathbf{x}), F_{2}^{+}(\mathbf{x})\right)=\mathbf{A x}+\mathbf{b}, \mathbf{x} \in \Sigma^{+} \\
\mathbf{F}^{-}(\mathbf{x})=\left(F_{1}^{-}(\mathbf{x}), F_{2}^{-}(\mathbf{x})\right)=\mathbf{A x}-\mathbf{b}, \mathbf{x} \in \Sigma^{-}
\end{array}\right.
$$

Notice that $\mathbf{F}(-\mathbf{x})=-\mathbf{F}(\mathbf{x})$, and so system (12) is odd symmetric.

\section{Equilibrium points and orbits}

The equilibrium points of system (10)-(11) with constant control signal $u=1$ or $u=-1$ are

$$
\overline{\mathbf{x}}^{ \pm}= \pm \overline{\mathbf{x}}= \pm\left(\begin{array}{c}
1+\frac{4 \beta \gamma^{2}}{m^{2}-\gamma^{2}} \\
\frac{2 \beta \gamma}{m^{2}-\gamma^{2}}
\end{array}\right),
$$

where $\overline{\mathbf{x}}^{+}=\overline{\mathbf{x}}$ and $\overline{\mathbf{x}}^{-}=-\overline{\mathbf{x}}$ correspond to $u=1$ and $u=-1$ respectively. The following remark addresses the admissibility of the two equilibrium, in accordance to the sign of the switching function $h(\overline{\mathbf{x}})=\bar{x}_{2}$. 
Remark 2. For system (10)-(11), the two equilibrium points $\overline{\mathbf{x}}^{ \pm}$, see (13), are admissible or real if $\beta>0$, non admissible or virtual if $\beta<0$ and boundary equilibrium points if $\beta=0$.

Orbits are well defined while they evolve without touching the switching manifold $\Sigma$. Then, let us consider an orbit $\mathbf{x}(\theta)$ through a point $\mathbf{x}(0)$. If $\mathbf{x}(0) \in \Sigma^{+}$and as long as $\mathbf{x}(\theta) \in \Sigma^{+}$, the orbit can be computed as a solution of the linear subsystem $d \mathbf{x} / d \theta=\mathbf{F}^{+}(\mathbf{x})$, that is

$$
\mathbf{x}(\theta)-\overline{\mathbf{x}}=\mathrm{e}^{\gamma \theta} \mathbf{M}(\theta ; m, \gamma)(\mathbf{x}(0)-\overline{\mathbf{x}}),
$$

where the matrix $\mathbf{M}$ is expressed depending on the modal parameter $m$ as

$$
\mathbf{M}(\theta ; m, \gamma)=\left\{\begin{array}{cc}
\left(\begin{array}{cc}
\cosh \theta-\gamma \sinh \theta & \left(\gamma^{2}-1\right) \sinh \theta \\
-\sinh \theta & \cosh \theta+\gamma \sinh \theta
\end{array}\right), & \text { if } m=1(Q<1 / 2), \\
\left(\begin{array}{cc}
1+\theta & \theta \\
-\theta & 1-\theta
\end{array}\right), & \text { if } m=0(Q=1 / 2), \\
\left(\begin{array}{cc}
\cos \theta-\gamma \sin \theta & \left(\gamma^{2}+1\right) \sin \theta \\
-\sin \theta & \cos \theta+\gamma \sin \theta
\end{array}\right), & \text { if } m=j(Q>1 / 2) .
\end{array}\right.
$$

Analogously, when $\mathbf{x}(0) \in \Sigma^{-}$, in the time interval including $\theta=0$ such that $\mathbf{x}(\theta) \in \Sigma^{-}$, the orbit is given by

$$
\mathbf{x}(\theta)+\overline{\mathbf{x}}=\mathrm{e}^{\gamma \theta} \mathbf{M}(\theta ; m, \gamma)(\mathbf{x}(0)+\overline{\mathbf{x}}) .
$$

\subsection{Sliding set and pseudo-equilibrium points}

In order to define the orbits at the discontinuity line $\Sigma$, we adopt the Filippov convex method, see for instance [27]. Regarding system (12), let first compute the product of the two normal components to the switching manifold, at a point $\mathbf{x}=\left(x_{1}, 0\right) \in \Sigma$,

$$
\left(\nabla h(\mathbf{x}) \mathbf{F}^{+}(\mathbf{x})\right) \cdot\left(\nabla h(\mathbf{x}) \mathbf{F}^{-}(\mathbf{x})\right)=F_{2}^{+}(\mathbf{x}) F_{2}^{-}(\mathbf{x})=x_{1}^{2}-1,
$$

where $\nabla(\cdot)$ is the gradient operator and

$$
\nabla h(\mathbf{x})=(0,1), \quad F_{2}^{+}(\mathbf{x})=-x_{1}+1, \quad F_{2}^{-}(\mathbf{x})=-x_{1}-1 .
$$

The crossing set $\Sigma^{c} \subset \Sigma$ is the set of all points $\mathbf{x} \in \Sigma$, where the normal components of $\mathbf{F}$ at both sides of the switching manifold have the same sign, that is

$$
\Sigma^{c}=\left\{\left(x_{1}, 0\right):\left|x_{1}\right|>1\right\} .
$$

At these points the orbits of system (12) cross the switching manifold $\Sigma$, i.e. the orbits reaching $\Sigma$ from one zone concatenate in a natural way with orbits leaving $\Sigma$ and entering the other zone.

The sliding set $\Sigma^{s} \subset \Sigma$ is the complement in $\Sigma$ of the crossing set $\Sigma^{c}$, i.e.

$$
\Sigma^{s}=\left\{\left(x_{1}, 0\right):\left|x_{1}\right| \leq 1\right\}
$$

which is the set of the points $\mathbf{x} \in \Sigma$, where the normal components of the vector fields to the discontinuity line have opposite sign or one of them vanishes. The sliding set is delimited by the two tangency points $\mathbf{x}_{B}^{ \pm}= \pm(1,0)$, and it is repulsive in forward time because for system (12),

$$
F_{2}^{+}(\mathbf{x}) \geq 0 \text { and } F_{2}^{-}(\mathbf{x}) \leq 0, \quad \mathbf{x} \in \Sigma^{s} .
$$

The Filippov method associates to every $\mathbf{x} \in \Sigma^{s}$ the so-called sliding vector field $\mathbf{F}^{s}(\mathbf{x})$ by means of the convex combination

$$
\mathbf{F}^{s}(\mathbf{x})=\lambda \mathbf{F}^{-}(\mathbf{x})+(1-\lambda) \mathbf{F}^{+}(\mathbf{x}),
$$

where $\lambda=\lambda(\mathbf{x})$ is selected so that $\mathbf{F}^{s}(\mathbf{x})$ is tangent to the sliding set. For system (10), $\lambda$ only depends on $x_{1}$ and its expression is given by

$$
\lambda\left(x_{1}\right)=\frac{F_{2}^{+}\left(x_{1}, 0\right)}{F_{2}^{+}\left(x_{1}, 0\right)-F_{2}^{-}\left(x_{1}, 0\right)}=\frac{1-x_{1}}{2} .
$$


Therefore, for $\left(x_{1}, 0\right) \in \Sigma^{s}$ we can define the scalar differential equation

$$
\frac{d x_{1}}{d \theta}=\lambda F_{1}^{-}\left(x_{1}, 0\right)+(1-\lambda) F_{1}^{+}\left(x_{1}, 0\right)=2 \beta \gamma x_{1} .
$$

Solutions of (16) are called sliding solutions. In particular, constant sliding solutions are called pseudo-equilibria of the system. When $\beta=0$, equation (16) reduces to $d x_{1} / d \theta=0$, and so every point in the sliding set is a pseudoequilibrium point of system (12). Paying attention only to the sliding dynamics, when $\beta \neq 0$, the origin is the only pseudo-equilibrium point, which is stable if $\beta>0$ and unstable if $\beta<0$. Since the sliding set $\Sigma^{s}$ is repulsive, it turns out that system (12) has a pseudo-saddle at the origin for $\beta>0$ and a repelling pseudo-node for $\beta<0$.

When $\beta>0$, the orbit of the vector field $\mathbf{F}^{+}$passing through the tangency point $\mathbf{x}_{B}^{+}$at a time, say $\theta=\theta_{B}$, belongs to $\Sigma^{+}$for $0<\left|\theta-\theta_{B}\right|<\varepsilon$, then the point $\mathbf{x}_{B}^{+}=(1,0)$ is a visible tangency point. Analogously, due to the symmetry of the vector field, the point $\mathbf{x}_{B}^{-}=-(1,0)$ is also a visible tangency point. Then, the only orbits entering the sliding set in forward time are those arriving tangentially to the two tangency points $\mathbf{x}_{B}^{ \pm}$and these orbits tend to the pseudo-saddle at the origin. In backward time, the sliding set is attractive and so sliding orbits arriving at the tangency points $\mathbf{x}_{B}^{ \pm}$ leave the sliding set.

When $\beta<0$, the orbit of the vector field $\mathbf{F}^{+}$through $\mathbf{x}_{B}^{+}$cannot belong to $\Sigma^{+}$for $0<\left|\theta-\theta_{B}\right|<\varepsilon$, then the point $\mathbf{x}_{B}^{+}$is an invisible tangency point and due to the symmetry, the point $\mathbf{x}_{B}^{-}$is also an invisible tangency point. In forward time, there is no orbit arriving to the tangency points, while in backward time all orbits reaching the sliding set remain therein, thus tending to the pseudo-node at the origin.

\subsection{Orbits through the switching manifold}

Although the system is discontinuous, it is possible to define a unique solution $\Phi\left(\theta, \mathbf{x}_{0}\right)$ with $\Phi\left(0, \mathbf{x}_{0}\right)=\mathbf{x}(0)$ for any initial condition $\mathbf{x}_{0}$, both in forward and backward time. Assuming $\mathbf{x}_{0} \in \Sigma^{+}$(the case $\mathbf{x}_{0} \in \Sigma^{-}$is symmetrical), the corresponding solution $\Phi\left(\theta, \mathbf{x}_{0}\right)$ can be computed by solving $d \mathbf{x} / d \theta=\mathbf{F}^{+}(\mathbf{x})$ while $\mathbf{x}(\theta) \in \Sigma^{+}$. If a forward time solution $\mathbf{x}(\theta)$ does not reach the switching manifold $\Sigma$, it tends to the stable equilibrium $\overline{\mathbf{x}}^{+}$. Otherwise, if the orbit reaches $\Sigma$ at the point $\left(x_{1}\left(\theta_{1}\right), 0\right)$, then necessarily $x_{1}\left(\theta_{1}\right) \geq 1$. If $x_{1}\left(\theta_{1}\right)>1$, then the orbit enters $\Sigma^{-}$and the vector field $\mathbf{F}^{-}$must be used to resume the computation. The case $x_{1}\left(\theta_{1}\right)=1$ is only possible when $\beta \geq 0$; if $\beta>0$, the orbit slides toward the pseudo-saddle equilibrium at the origin, but if $\beta=0$, then $\mathbf{x}(\theta)=\mathbf{x}\left(\theta_{1}\right)=(1,0)$ for all $\theta>\theta_{1}$.

In contrast, if a backward time solution $\mathbf{x}(\theta)$ satisfying $x(0) \in \Sigma^{+}$reaches the discontinuity line $\Sigma$, then there exists a time $\theta_{2}<0$ for which $\mathbf{x}\left(\theta_{2}\right)=\left(x_{1}\left(\theta_{2}\right), 0\right) \in \Sigma$, with $x_{1}\left(\theta_{2}\right) \leq 1$. At this point different cases arise when $\theta<\theta_{2}$. If $x_{1}\left(\theta_{2}\right)<-1$, the orbit crosses $\Sigma$ and $\mathbf{F}^{-}$must be used to resume the reverse time computation. When $-1 \leq x_{1}\left(\theta_{2}\right) \leq 1$, then the following cases arise:

(a) If $x_{1}\left(\theta_{2}\right)=0$, then $\mathbf{x}(\theta)=\mathbf{x}\left(\theta_{2}\right)=(0,0)$ for all $\theta<\theta_{2}$.

(b) If $\beta<0$, then the orbit slides in backward time towards the pseudo-equilibrium point at the origin.

(c) If $\beta=0$, then $\mathbf{x}(\theta)=\mathbf{x}\left(\theta_{2}\right)$ for all $\theta<\theta_{2}$.

(d) If $\beta>0$, then the following cases arise

(d1) If $x_{1}\left(\theta_{2}\right)=-1$, then the orbit crosses $\Sigma$ and then follows in backward time the unique standard orbit in $\Sigma^{-}$ through the point $\mathbf{x}_{B}^{-}$.

(d2) If $-1<x_{1}\left(\theta_{2}\right)<0$, then the orbit slides in backward time towards the point $\mathbf{x}_{B}^{-}$. Then, we proceed as in (d1).

(d3) If $x_{1}\left(\theta_{2}\right)=1$, then the orbit crosses $\Sigma$ and then follows in backward time the unique standard orbit in $\Sigma^{+}$ through the point $\mathbf{x}_{B}^{+}$.

(d4) If $0<x_{1}\left(\theta_{2}\right)<1$, then the orbit slides in backward time towards the point $\mathbf{x}_{B}^{+}$. Then, we proceed as in (d3).

The different situations defined above are illustrated in Fig. 3 when the equilibria of system (12) are foci. Observe that, excepting the orbits tangent to the switching manifold $\Sigma^{s}$ at the points $\mathbf{x}_{B}^{ \pm}=( \pm 1,0)$, each panel can be obtained from the other one by reversing the sign of the arrows. Note also that the solutions of the system are not invertible in the classical sense, since the orbits can overlap on the sliding set, see [27]. 


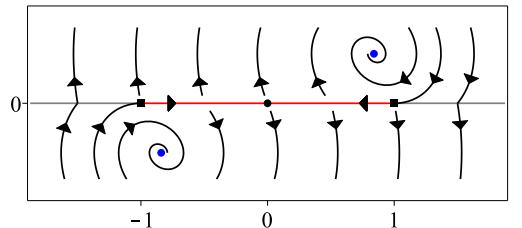

(a) $\beta>0$ and forward time

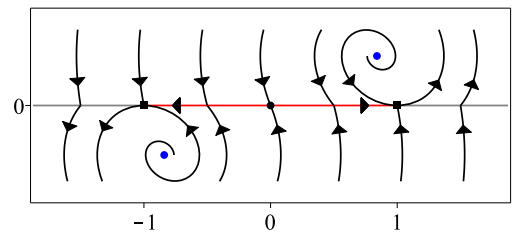

(d) $\beta>0$ and backward time

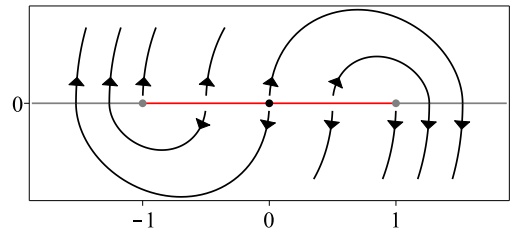

(b) $\beta=0$ and forward time

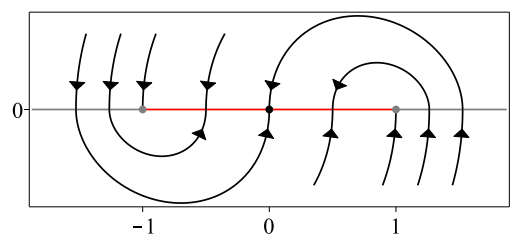

(e) $\beta=0$ and backward time

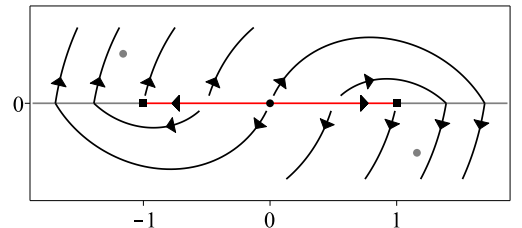

(c) $\beta<0$ and forward time

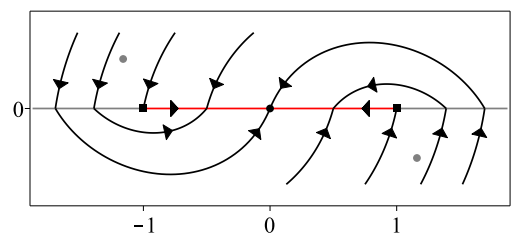

(f) $\beta<0$ and backward time

Figure 3. Orbits in a neighborhood of the sliding set when the equilibria are foci. Upper and lower panels stand for forward and backward time respectively. Left, central and right panels do for $\beta>0, \beta=0$ and $\beta<0$ respectively. The red segment corresponds to the sliding set $\Sigma^{s}$, bounded by $\mathbf{x}_{B}^{ \pm}$points, represented as boxed points, and the circled points stand for equilibria, in blue color if they are admissible, otherwise in gray color.

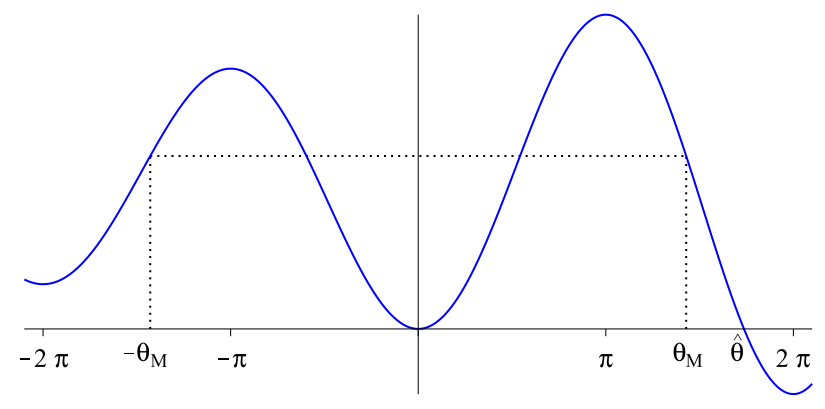

Figure 4. Graph of function $\varphi_{-\gamma}(\theta)$ with $\gamma<0$, including the value $\hat{\theta}$ defined by $\varphi_{-\gamma}(\hat{\theta})=0$ with $\pi<\hat{\theta}<2 \pi$ and the value $\theta_{M}$ defined by $\varphi_{-\gamma}\left(-\theta_{M}\right)=\varphi_{-\gamma}\left(\theta_{M}\right)$ with $\pi<\theta_{M}<\hat{\theta}$.

\section{Main results}

Recall that system (7)-(8) is symmetrical with respect to the origin. Consequently, if $\gamma_{1}(\theta)=\Phi\left(\theta, \mathbf{x}_{0}\right)$ is a solution with $\gamma_{1}(0)=\mathbf{x}_{0}$, then $\gamma_{2}(\theta)=-\Phi\left(\theta, \mathbf{x}_{0}\right)$ is also a solution with $\gamma_{2}(0)=-\mathbf{x}_{0}$.

Invariant closed curves containing points in the sliding set $\Sigma^{s}$ are called sliding closed curves. Since orbits do not arrive at the repulsive sliding set in forward time, the only possible sliding closed curves occur in backward time. If $\beta=0$, from (16) every point in the sliding set is a pseudo-equilibrium point and when $\beta<0$ the origin attracts all the sliding orbits. Consequently, sliding closed curves cannot exist when $\beta \leq 0$. The existence of sliding closed curves for $\beta>0$ will be analyzed later.

On the other hand, the absolute value of the $x_{1}$-intersection of a possible crossing limit cycle with the switching manifold is greater or equal than 1 . When the quoted absolute value is 1 , the limit cycle links the two endpoints $\mathbf{x}_{B}^{+}$and $\mathbf{x}_{B}^{-}$of the sliding set. This cycle is called critical crossing limit cycle (CC limit cycle, for short) and it corresponds to the transition from a sliding invariant closed curve to a crossing limit cycle, being a symmetric instance of the generic case studied in [28].

In order to state the main results concerning the existence of limit cycles when the equilibria are real foci, that is 
$\beta>0$ and $Q>1 / 2$, we resort to several functions, see [22] for more details. First, we consider the auxiliary function $\varphi_{\gamma}(\theta)$ introduced in [26]

$$
\varphi_{\gamma}(\theta)=1-e^{\gamma \theta}(\cos \theta-\gamma \sin \theta) .
$$

This function has local maxima at $\theta=(2 n-1) \pi$ and local minima at $\theta=2 n \pi$, where $n \in \mathbb{N}$. We denote as $\hat{\theta} \in(\pi, 2 \pi)$ the minimum value positive such that

$$
\varphi_{-\gamma}(\hat{\theta})=1-e^{-\gamma \hat{\theta}}(\cos \hat{\theta}+\gamma \sin \hat{\theta})=0 .
$$

Also, we introduce the positive function $\beta_{h c}(\gamma)$ defined for $\gamma<0$ as follows,

$$
\beta_{h c}(\gamma)=\frac{1}{2}+\frac{\cot \hat{\theta}}{2 \gamma}=\frac{e^{\gamma \hat{\theta}}}{2 \gamma \sin \hat{\theta}},
$$

where $\hat{\theta}$ is defined in (18). For the sake of convenience, let us introduce the function

$$
\beta_{c c}(\gamma)=2 \beta_{h c}(\gamma) \text {. }
$$

Moreover, we introduce the value $\theta_{M}$, which is the only root of equation

$$
\gamma \operatorname{coth}\left(\gamma \theta_{M}\right)-\cot \theta_{M}=0,
$$

in the interval $(\pi, 3 \pi / 2)$. This comes from the condition $\varphi_{-\gamma}\left(-\theta_{M}\right)=\varphi_{-\gamma}\left(\theta_{M}\right)$ with $\pi<\theta_{M}<\hat{\theta}$. Then, we define the associated positive function

$$
\beta_{s n}(\gamma)=\frac{\left(1+\gamma^{2}\right) \sin \theta_{M}}{2 \gamma\left(\gamma \sin \theta_{M}-\sinh \left(\gamma \theta_{M}\right)\right)} .
$$

The function $\varphi_{-\gamma}(\theta)$ and corresponding values $\hat{\theta}$ an $\theta_{M}$ are represented in Figure 4 for $\gamma<0$.

Finally, taking $\gamma=\gamma(Q)=-\left(4 Q^{2}-1\right)^{-1 / 2}<0$, and by using the notation $\beta(Q)=\beta(\gamma(Q))$, we state our main result.

Proposition 3. System (7)-(8) has always a pseudo-equilibrium point at the origin and the following statements are true.

(a) If $\beta<0$, there are two virtual equilibria, one stable crossing limit cycle and there are no sliding closed curves.

(b) If $\beta=0$, every point in the sliding set is a pseudo-equilibrium point and there are no sliding closed curves. Moreover, if $Q \leq 1 / 2$, there are no crossing limit cycles and when $Q>1 / 2$ there is one crossing limit cycle which is stable.

(c) If $\beta>0$, there are two real equilibria. Moreover, if $Q \leq 1 / 2$, there are no periodic orbits and when $Q>1 / 2$, there exist three positive functions $\beta_{h c}, \beta_{c c}, \beta_{s n}$, defined in (19), (20), (22), respectively, such that $0<\beta_{h c}(Q)<$ $\beta_{c c}(Q)<\beta_{s n}(Q)$, and the following statements hold.

(c1) If $0<\beta<\beta_{h c}(Q)$, there exist one stable crossing limit cycle and two unstable sliding limit cycles.

(c2) If $\beta=\beta_{h c}(Q)$, there exist one stable crossing limit cycle and two homoclinic connections to the origin.

(c3) If $\beta_{h c}(Q)<\beta<\beta_{c c}(Q)$, there exist one stable crossing limit cycle and one unstable sliding limit cycle.

(c4) If $\beta=\beta_{c c}(Q)$, there exist one stable crossing limit cycle and one unstable critical crossing limit cycle.

(c5) If $\beta_{c c}(Q)<\beta<\beta_{s n}(Q)$, there exist two crossing limit cycles with opposite stability.

(c6) If $\beta=\beta_{s n}(Q)$, there exists one crossing limit cycle which is semi-stable.

(c7) If $\beta>\beta_{s n}(Q)$, there are no crossing limit cycles.

Note that the crossing limit cycles (stable or not) always surround the sliding set $\Sigma^{S}$. Furthermore, sliding closed curves are unstable and not observable in practice but, since they determine the attraction basin of the stable equilibrium points, become of paramount relevance.

The complete bifurcation set of system (7)-(8) in the parameter plane $(Q, \beta)$ is shown in Fig. 5. We see that from the point $(1 / 2,0)$, five codimension one bifurcation lines emerge. These critical lines split the parameter plane in regions with different dynamical behavior. The corresponding transitions are described following a clockwise direction as follows. 


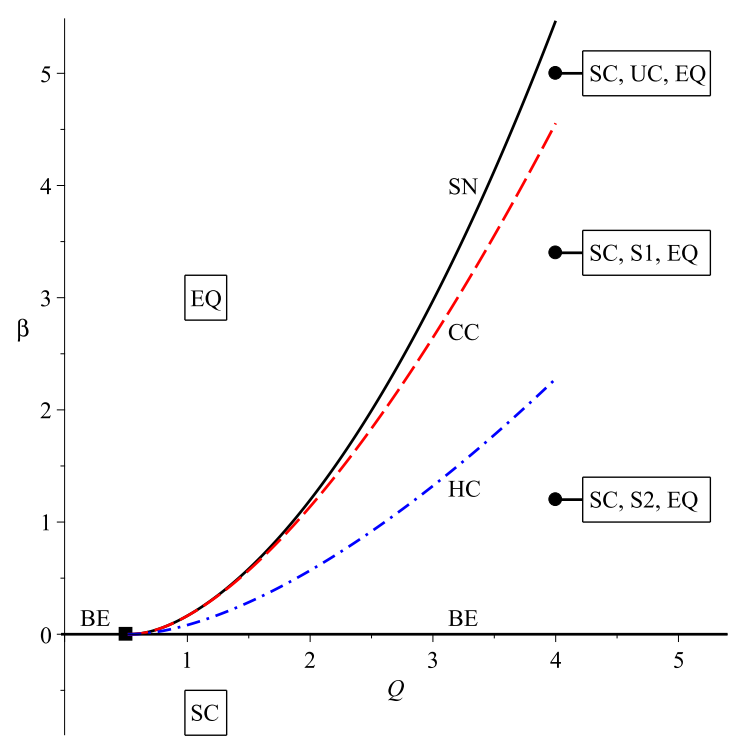

Figure 5. Complete bifurcation set in the parameter plane $(Q, \beta)$. Note the existence of a codimension-2 bifurcation point $(Q=1 / 2, \beta=0)$ organizing the five codimension-1 bifurcation lines. Following a clockwise direction, these critical lines are: (BE) boundary equilibrium bifurcation ( $\beta=0$ with $Q<1 / 2$, in solid black), (SN) smooth saddle-node bifurcation for cycles (upper solid black curve), (CC) critical crossing cycle bifurcation (dashed red curve), (HC) homoclinic connection bifurcation (dash-dotted blue curve) and again (BE) boundary equilibrium bifurcation ( $\beta=0$, but with $Q>1 / 2$ ), now involving small sliding limit cycles. Acronyms for valid limit sets in the regions between those curves stand for: EQ (equilibria), SC (stable crossing cycle), UC (unstable crossing cycle), S1 (single unstable sliding cycle) and S2 (twin unstable sliding cycles).

(a) The segment

$$
B E^{-}=\{(Q, \beta): 0<Q<1 / 2, \beta=0\},
$$

where a continuum of pseudo-equilibrium points exists and a boundary equilibrium bifurcation occurs. The two virtual equilibria existing for $\beta<0$ become two real equilibria for $\beta>0$ and also, the stable limit cycle existing for $\beta<0$ collapses at $\beta=0$, with a double heteroclinic connection between the tangency points $\mathbf{x}_{B}^{ \pm}$and disappears for $\beta>0$. Details of this configuration appear Fig. 6. This bifurcation has never been reported before up to the best of our knowledge.

(b) The line

$$
S N=\left\{(Q, \beta): \beta=\beta_{S n}(Q)\right\},
$$

where one semi-stable crossing limit cycle exists and a saddle node bifurcation occurs. The two crossing limit cycles with opposite stabilities existing for $\beta_{c c}(Q)<\beta<\beta_{s n}(Q)$ disappear for $\beta>\beta_{s n}(Q)$.

(c) The line

$$
C C=\left\{(Q, \beta): \beta=\beta_{c c}(Q)\right\},
$$

where a critical crossing limit cycle exists and a critical crossing bifurcation occurs. The sliding limit cycle existing for $\beta_{h c}(Q)<\beta<\beta_{c c}(Q)$ becomes a crossing limit cycle for $\beta_{c c}(Q)<\beta<\beta_{s n}(Q)$.

(d) The line

$$
H C=\left\{(Q, \beta): \beta=\beta_{h c}(Q)\right\},
$$

where a double homoclinic connection to the origin exists giving rise to a homoclinic bifurcation. The two mutually symmetric sliding limit cycles existing for $0<\beta<\beta_{h c}(Q)$ become one sliding limit cycle, which surrounds the pseudo-saddle at the origin for $\beta>\beta_{h c}(Q)$.

(e) The segment

$$
B E^{+}=\{(Q, \beta): Q>1 / 2, \beta=0\}
$$

where a continuum of pseudo-equilibrium points exists and a boundary equilibrium bifurcation occurs. The two virtual equilibria existing for $\beta<0$ become real for $\beta>0$, each one surrounded by one sliding limit cycle.

The following section is devoted to prove Proposition 3. 


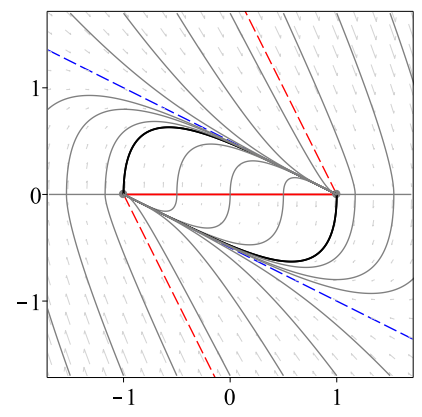

Figure 6. Critical configuration for $\beta=0$, while $Q<1 / 2$. Note that from each pseudo-equilibrium point of the sliding set, two heteroclinic orbits emanate, one to $x_{B}^{+}=(1,0)$ and the other to $x_{B}^{-}=(-1,0)$. In particular, the two largest heteroclinic orbits form a closed path joining these singular points by a double heteroclinic connection.

\section{The self oscillating dynamics}

Recall that invariant closed curves containing points in the sliding set $\Sigma^{s}$ are called sliding closed curves. In our case, due to the repulsive character of $\Sigma^{s}$ and from (16), the only possible sliding closed curves occur in backward time and can only exist if $\beta>0$.

Here, we are in position to establish our first result dealing with the non oscillatory dynamics.

Proposition 4. (The case of real or boundary equilibria with real eigenvalues) If system (12) has real or boundary equilibria with associated real eigenvalues then there are neither crossing periodic orbits nor invariant closed curves.

Proof. If the matrix of our system has real eigenvalues, then the equilibria have associated some invariant manifolds which cannot be crossed by the orbits evolving around each equilibrium. As a consequence, any arbitrary trajectory at most can have one switching and the conclusion follows.

Remark 3. We remark that the hypotheses of Proposition 4 are only satisfied when $\beta \geq 0$, and also, $0<Q \leq 1 / 2$ if we model our system with equations (7)-(8), or $m \neq j$ if equations (10)-(11) are used.

\subsection{The Poincaré map}

In order to study the existence of crossing periodic orbits, we introduce a Poincare map associated to the switching manifold $\Sigma$. Take $\left(x_{1}(0), x_{2}(0)\right)=\left(z_{1}, 0\right)$, with $z_{1} \geq 1$ as initial point of an orbit and integrate backwards. If the orbit eventually reaches the switching line $\Sigma$ at a point $(z, 0)$, then $z<1$ and a Poincaré map $P$ can be defined as $z_{1}=P(z)$. Obviously, variables $z$ and $P(z)$ also depend on variables $\beta$ and $\gamma$, but this functional dependence only will be made explicit when it is needed.

The direction of the flow assures that orbits starting at the points $\left(z_{1}, 0\right)$ with $z_{1}>1$, go in forward time into the zone $\Sigma^{-}$. If the orbit reaches $\Sigma$ again at a point $\left(z_{2}, 0\right)$ then $z_{2}<-1$, and we can define another Poincaré map $\widetilde{P}$ as $z_{2}=\widetilde{P}\left(z_{1}\right)$, for $z_{1}>1$.

It is direct to see that when $(\widetilde{P} \circ P)(z)=z$, a crossing limit cycle exists and so, the crossing limit cycles correspond to fixed points of the map $\widetilde{P} \circ P$.

Assuming that $P(z)=z_{1}$, we realize that the symmetry of the system imposes that $\widetilde{P}(-z)=-z_{1}$, and so

$$
(\widetilde{P} \circ P)(z)=-P(-P(z))=(-P) \circ(-P)(z) .
$$

Consequently, the limit cycles correspond with the points $\bar{z}<-1$ such that $P(\bar{z})=-\bar{z}$.

It is worth noting that map $P$ is defined for $-\infty<z<-1$ and it monotonically decreases on its domain. Moreover, the derivative of the full Poincare map $\widetilde{P} \circ P$ at a fixed point $\bar{z}$ is

$$
(\widetilde{P} \circ P)^{\prime}(\bar{z})=-P^{\prime}(-P(\bar{z}))\left(-P^{\prime}(\bar{z})\right)=\left(-P^{\prime}(\bar{z})\right)^{2},
$$




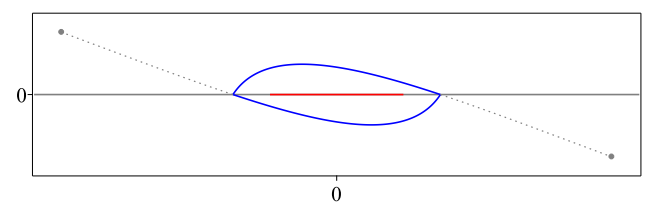

Figure 7. Limit cycle in the phase plane (the blue closed curve) for the virtual equilibria $(\beta<0)$ with real eigenvalues $(Q<1 / 2)$. The red segment is the sliding subset of the switching manifold $\left(\Sigma^{s} \subset \Sigma\right)$ and the dotted lines stand for non admissible orbits approaching the virtual equilibria.

hence, the fixed point is stable when $\left|P^{\prime}(\bar{z})\right|<1$ and unstable when $\left|P^{\prime}(\bar{z})\right|>1$.

In looking for oscillating behavior and according to Remark 3, we only have to consider the Poincaré map in the case of real or boundary foci $(\beta \geq 0$ with $m=j)$ and in the case of virtual equilibria $(\beta<0)$.

Proposition 5. (The case of boundary foci) If system (12) has boundary equilibria with complex eigenvalues, then there is one periodic orbit which is stable.

Proof. The system has boundary equilibria whenever $\beta=0$. The boundary equilibrium corresponding to the vector field $\mathbf{F}^{+}$is located at the point $(1,0)$. If the eigenvalues are complex, then $m=j$. Taking $m=j$ in (14), it is deduced that the orbit from any point $(z, 0) \in \Sigma$, with $z<1$, to the point $(P(z), 0) \in \Sigma$, with $z>1$, takes a flight time $\theta=\pi$. Then, also from (14), the Poincaré map $P$ is explicitly obtained

$$
P(z)=1-e^{\gamma \pi}(z-1), \quad z<1 .
$$

The equation $z=-P(z)$ has a unique solution $\bar{z}=\operatorname{coth}\left(\frac{\gamma \pi}{2}\right)<-1$. Moreover, the modulus of the derivative of Poincaré map is $\left|P^{\prime}(z)\right|=e^{\gamma \pi}<1$. Hence, the system has only one crossing limit cycle, which is stable.

Remark 4. We remark that the hypotheses of Proposition 5 are only satisfied when $\beta=0$ and $Q>1 / 2$.

Proposition 6. (The case of virtual equilibria) If the equilibria of system (12) are virtual then there is one limit cycle which is stable.

Proof. The system has virtual equilibria whenever $\beta<0$. To determine the existence of limit cycles, we consider the solution of equation $P(z)=-z$, or equivalently the zeroes of the function

$$
g(z)=P(z)+z
$$

Next, we study the function $g$ by considering the Poincare map $P$ taking into account the three different types of dynamics possible in system (12): node, improper node and focus.

(a) If the virtual equilibria are of node type, then $\gamma<-1$ and by solving (14) taking $m=1$, the following parametric representation of the Poincaré map $z_{1}(\theta)=P(z(\theta))$ is obtained,

$$
z(\theta)=1-\frac{2 \beta \gamma}{\gamma^{2}-1} \frac{e^{-\gamma \theta}+\gamma \sinh \theta-\cosh \theta}{\sinh \theta}, \quad z_{1}(\theta)=1+\frac{2 \beta \gamma}{\gamma^{2}-1} \frac{e^{\gamma \theta}-\gamma \sinh \theta-\cosh \theta}{\sinh \theta}, \quad 0<\theta<\infty,
$$

and so

$$
g(z)=g(z(\theta))=2-\frac{4 \beta \gamma}{\gamma^{2}-1}\left(\gamma-\frac{\sinh (\gamma \theta)}{\sinh \theta}\right) .
$$

Noting that $\lim _{\theta \rightarrow 0} z(\theta)=1$ and $\lim _{\theta \rightarrow \infty} z(\theta)=-\infty$, we get

$$
\lim _{z \rightarrow 1} g(z)=\lim _{\theta \rightarrow 0} g(z(\theta))=2, \quad \lim _{z \rightarrow-\infty} g(z)=\lim _{\theta \rightarrow \infty} g(z(\theta))=-\infty .
$$

Furthermore, the derivative of the function $g$ with respect to variable $\theta$ is

$$
\frac{d g}{d \theta}=\frac{4 \beta \gamma(\gamma \sinh \theta \cosh (\gamma \theta)-\cosh \theta \sinh (\gamma \theta))}{\left(\gamma^{2}-1\right) \sinh ^{2} \theta}<0 .
$$

The limit cycle corresponding to this case case is represented in Fig. 7. 
(b) If the virtual equilibria are improper nodes, by solving (14) when $m=0$ we get

$$
z(\theta)=1-2 \beta \frac{1+\theta-e^{\theta}}{\theta}, \quad z_{1}(\theta)=1+2 \beta \frac{1-\theta-e^{-\theta}}{\theta}, \quad 0<\theta<\infty,
$$

and so,

$$
g(z(\theta))=2-4 \beta\left(1-\frac{\sinh \theta}{\theta}\right)
$$

Note that

$$
\lim _{z \rightarrow 1} g(z)=\lim _{\theta \rightarrow 0} g(z(\theta))=2, \quad \lim _{z \rightarrow-\infty} g(z)=\lim _{\theta \rightarrow \infty} g(z(\theta))=-\infty .
$$

Furthermore, the derivative of the function $g$ with respect to variable $\theta$ is

$$
\frac{d g}{d \theta}=\frac{4 \beta(\theta \cosh \theta-\sinh \theta)}{\theta^{2}}<0 .
$$

(c) If the virtual equilibria are foci, by solving (14) taking $m=j$, we have

$$
z(\theta)=1-\frac{2 \beta \gamma}{\gamma^{2}+1} \frac{e^{-\gamma \theta}+\gamma \sin \theta-\cos \theta}{\sin \theta}, \quad z_{1}(\theta)=1+\frac{2 \beta \gamma}{\gamma^{2}+1} \frac{e^{\gamma \theta}-\gamma \sin \theta-\cos \theta}{\sin \theta}, \quad 0<\theta<\pi .
$$

and then

$$
g(z)=g(z(\theta))=2-\frac{4 \beta \gamma}{\gamma^{2}+1}\left(\gamma-\frac{\sinh (\gamma \theta)}{\sin \theta}\right) .
$$

In this case

$$
\lim _{z \rightarrow 1} g(z(\theta))=\lim _{\theta \rightarrow 0} g(z(\theta))=2, \quad \lim _{z \rightarrow-\infty} g(z(\theta))=\lim _{\theta \rightarrow \pi} g(z(\theta))=-\infty,
$$

and the derivative of function $g$ with respect to the variable $\theta$ is

$$
\frac{d g}{d \theta}=\frac{4 \beta \gamma(\gamma \sin \theta \cosh (\gamma \theta)-\cos \theta \sin (\gamma \theta))}{\left(\gamma^{2}+1\right) \sin ^{2} \theta}<0 .
$$

In the three cases, the function $z(\theta)$ is decreasing and function $z_{1}(\theta)$ is increasing on its respective domains, that is $d z / d \theta<0$ and $d z_{1} / d \theta>0$. Consequently,

$$
\frac{d P}{d z}=\frac{d z_{1} / d \theta}{d z / d \theta}<0,
$$

and also,

$$
\frac{d g}{d \theta}=\left(\frac{d g}{d z}\right)\left(\frac{d z}{d \theta}\right)<0,
$$

and then $d g / d z>0$. Since $\lim _{z \rightarrow-\infty} g(z)=-\infty$, and $\lim _{z \rightarrow 1} g(z)=2$, and taking into account that the function $g$ is increasing with $z$, we conclude that there exists only one value $\bar{z}$ with $g(\bar{z})=0$, and system (12) has only one limit cycle. Furthermore, from (23) it follows

$$
0<\frac{d g}{d z}=\frac{d P}{d z}+1,
$$

hence the inequalities $-1<d P / d z<0$ are satisfied and the limit cycle is stable.

It remains to consider the dynamics when the equilibria are real foci. This case was thoroughly studied in [22]. For the sake of completeness, the main results concerning this case are summarized in the two following propositions, whose proofs appear in [22]. Herein, the parameter $Q>1 / 2$ is assumed constant, $\beta>0$ is the bifurcation parameter and functions $\beta_{h c}, \beta_{c c}$ and $\beta_{s n}$ are defined in (19), (20) and (22) respectively. Proposition 7 deals with sliding closed curves and Proposition 8 does with crossing limit cycles. Also, phase portraits for the dynamics in some regions of the parameter plane, which are defined in the quoted propositions, are represented in Fig. 8.

Proposition 7. (Sliding limit cycles for real foci) Assuming $\gamma<0$ in system (12) and taking $\beta_{h c}(\gamma)$ as in (19), the following statements hold 


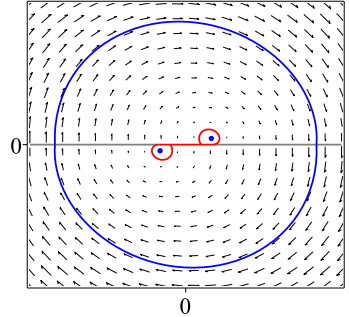

(a) $0<\beta<\beta_{h c}(Q)$

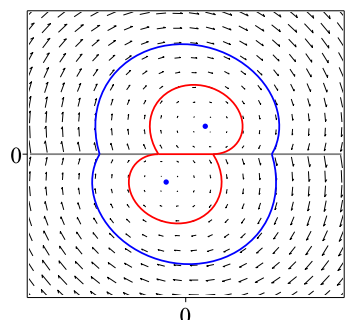

(d) $\beta=\beta_{c c}(Q)$

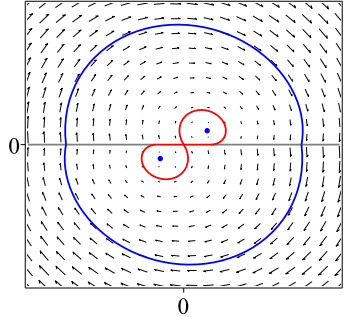

(b) $\beta=\beta_{h c}(Q)$

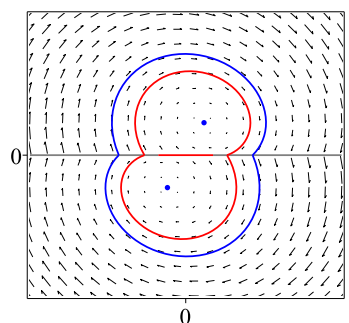

(e) $\beta_{c c}(Q)<\beta<\beta_{s n}(Q)$

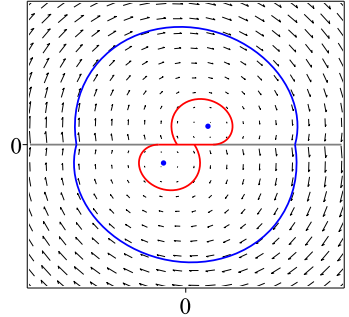

(c) $\beta_{h c}(Q)<\beta<\beta_{c c}(Q)$

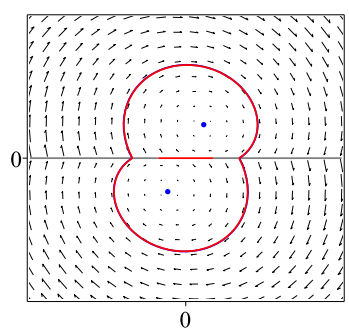

(f) $\beta=\beta_{s n}(Q)$

Figure 8. Limit sets in the phase plane corresponding to the different dynamic scenarios in Propositions 7-8. Parameter $Q>1 / 2$ is fixed and parameter $\beta$ is specified in the caption.

(a) The function $\beta_{h c}(\gamma)$ is increasing with $\gamma$.

(b) If $0<\beta<\beta_{h c}(\gamma)$, then there exists a symmetric pair of unstable sliding limit cycles, each one living in only one zone.

(c) If $\beta=\beta_{h c}(\gamma)$, then the above limit cycles become a symmetric pair of unstable sliding homoclinic connections to the origin.

(d) If $\beta_{h c}(\gamma)<\beta<2 \beta_{h c}(\gamma)$, then there exists one unstable sliding limit cycle being symmetrical with respect to the origin.

Proposition 8. (Crossing limit cycles for real foci) Assuming $\gamma<0$ in system (12), taking $\beta_{s n}(\gamma)$ as in (22) and $\beta_{c c}(\gamma)=2 \beta_{h c}(\gamma)$, see (20), the following statements hold.

(a) The functions $\beta_{s n}(\gamma)$ and $\beta_{c c}(\gamma)$ are positive and increasing with $\gamma$.

(b) If $\beta>\beta_{s n}(\gamma)$, then there are no crossing limit cycles.

(c) If $\beta=\beta_{s n}(\gamma)$, then there exists one semi-stable crossing limit cycle.

(d) If $\beta_{c c}<\beta<\beta_{s n}(\gamma)$, then there exist two crossing limit cycles. The inner one is unstable being the outer one stable.

(e) If $\beta=\beta_{c c}(\gamma)$, then there exist one unstable critical crossing limit cycle and one stable crossing limit cycle.

(f) If $\beta<\beta_{c c}(\gamma)$, then there exists one stable crossing limit cycle.

Finally, the proof of Proposition 3 follows from propositions 4, 5, 6, 7 and 8.

Table 1. Circuit parameters

\begin{tabular}{|c|c|c|c|c|c|}
\hline$V_{g}$ & $L$ & $r_{l s}$ & $C$ & $r_{c s}$ & $r_{c p}$ \\
\hline $10 \mathrm{~V}$ & $10 \mu \mathrm{H}$ & $0.2 \Omega$ & $10 \mathrm{nF}$ & $0.1 \Omega$ & $1 \mathrm{G} \Omega$ \\
\hline
\end{tabular}




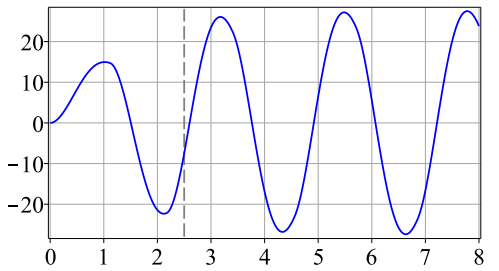

(a) $v_{o}$ versus time $(\mu \mathrm{s})$

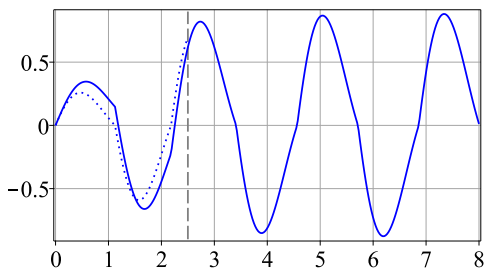

(d) $i_{L}$ and $h_{s}(t)$ versus time $(\mu \mathrm{s})$

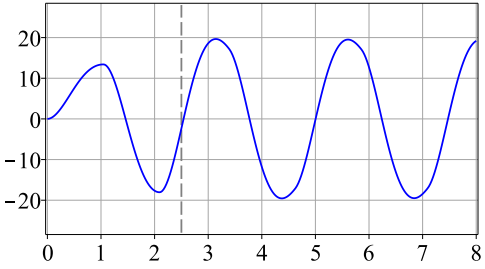

(b) $v_{o}$ versus time $(\mu \mathrm{s})$

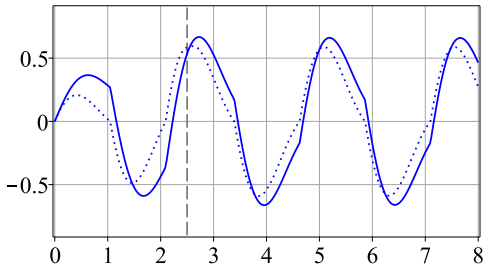

(e) $i_{L}$ and $h_{s}(t)$ versus time $(\mu \mathrm{s})$

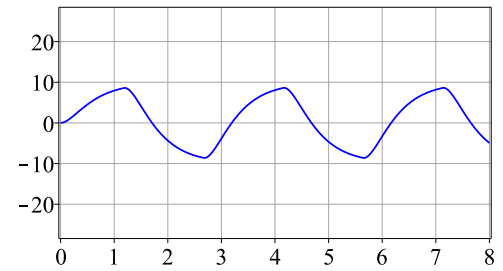

(c) $v_{o}$ versus time $(\mu \mathrm{s})$

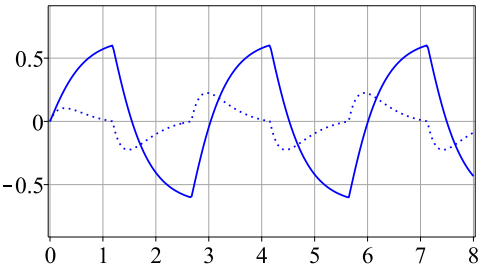

(f) $i_{L}$ and $h_{s}(t)$ versus time $(\mu \mathrm{s})$

Figure 9. Dynamical response for the parallel topology $\left(R_{o s}=0\right)$. Parameters are those in Table 1 and the value of $R_{o p}$ is selected using the values $75 \Omega, 50 \Omega$ or $15 \Omega$ for the left, central or right panels, respectively. Notice that these parameters correspond to the quality factors $Q_{1}=2.3241$, $Q_{2}=1.5624$ and $Q_{3}=0.4769$. The output voltage $v_{o}(t)$ (in V) is represented in the top panels. The inductor current $i_{L}(t)$ and the control current $h_{s}(t)=i_{L}(t)-\left(a_{v} / a_{i}\right) v_{o}(t)$ (both in A) are represented in the bottom panels using continuous and dotted lines respectively. Using $t_{S}=2.5 \mu \mathrm{s}$, the relation $\left(a_{v} / a_{i}\right)$ has been selected as follows: $a_{v} / a_{i}=10 \mathrm{mV} / \mathrm{A}$ if $0<t<t_{S}$ and $a_{v} / a_{i}=0$ if $t>t_{S}$ for the left panels; $a_{v} / a_{i}=20 \mathrm{mV} / \mathrm{A}$ for $0<t<t_{S}$ and $a_{v} / a_{i}=10 \mathrm{mV} / \mathrm{A}$ if $t>t_{S}$ for the central panels; and $a_{v} / a_{i}=70 \mathrm{mV} / \mathrm{A}$ for the right panels. Notice that the switching action is produced when $h_{s}(t)=0$, see the dotted lines in the bottom panels.

\section{Some applications including the voltage feedback term}

Although a bare ZCS control, that is with $a_{v}=0$, has the advantage of minimizing the stress in the switching devices, the addition of a voltage feedback term $\left(a_{v} \neq 0\right)$, can be of interest in some applications, as it can be seen in the cases detailed below.

\subsection{Control with a low quality factor of the resonator}

In the parallel topology of the resonant inverter under ZCS control, that is with $R_{o s}=0$ and $a_{v}=0$, the parameter $\beta$ is lower and close to 1 . In particular, $\beta=1$ for ideal LC resonator components, and consequently, there are some critical values of the quality factor $Q_{h c}$ and $Q_{s n}$ such that $\beta_{h c}\left(Q_{h c}\right)=1$ and $\beta_{s n}\left(Q_{s n}\right)=1$. Numerical computations show that the critical values for the homoclinic connection and the saddle-node bifurcations are $Q_{h c}=2.6075$ and $Q_{s n}=1.8552$, respectively. Then, for values of the parameter $Q<Q_{h c}$, the origin in the phase plane belongs to the attraction basin of equilibria, see Fig. 8(c-e), so that orbits starting at the origin will address to one of the two undesired equilibria, thus precluding the oscillatory dynamics. From the analysis made in this paper, it becomes clear that by varying the gain control parameter $a_{v}$, the value of $g_{C}$ can be adjusted, see (1), in order to reduce the parameter $\beta$, as much as desired, even making it negative, according to (6). Then, by forcing $\beta<\beta_{h c}(Q)$, the oscillatory dynamics can be guaranteed under a standard start up of the circuit with zero initial conditions. Moreover, if $Q_{s n}<Q<Q_{h c}$, the voltage feedback can be active only for a short time, just to avoid the dynamics going to one equilibrium. After that, the normal ZCS control can be reestablished.

The following three examples correspond to a parallel resonant inverter with a choice of three values of the quality factor $Q \in\left\{Q_{1}, Q_{2}, Q_{3}\right\}$, such that $Q_{s n}<Q_{1}<Q_{h c}, 1 / 2<Q_{2}<Q_{s n}$ and $Q_{3}<1 / 2$. Note that $Q_{1}>Q_{2}>Q_{3}$. Then, different actions in the control, in order to ensure the oscillatory dynamics in the steady state, are detailed below.

(a) The first example corresponds to a circuit with components values in Table 1, and parallel topology $\left(R_{o s}=0\right)$ with $R_{o p}=75 \Omega$, thus corresponding to the quality factor $Q_{1}=2.3241$, with the associated critical values $\beta_{h c}=0.7880$, $\beta_{c c}=1.5760$ and $\beta_{s n}=1.6931$. In the two diagrams on the left of Fig. 9, the time evolution of the output voltage 
$v_{o}$ (top panel) and the inductor currents $i_{L}$ and the control signal $h_{s}$ (bottom panel) are shown. The initial state of the dynamics is the origin in the phase plane, and it can be observed that the steady state is nearly reached in a few cycles. The mark at the time $t_{S}=2.5 \mu \mathrm{s}$ indicates a transition in the voltage-current feedback, such that either $a_{v} / a_{i}=10 \mathrm{mV} / \mathrm{A}$ if $0<t<t_{S}$ or $a_{v} / a_{i}=0$ if $t>t_{S}$. Notice that while $t<t_{S}$, the value $a_{v} / a_{i}=10 \mathrm{mV} / \mathrm{A}$ produces the parameter $\beta=0.2447<\beta_{h c}\left(Q_{1}\right)=0.7880$, so that the dynamics from the origin will evolve to the limit cycle, instead of the equilibrium. Furthermore, considering these circuit parameters and ZCS control, it is found that $\beta=0.9780<\beta_{s n}\left(Q_{1}\right)=1.6931$. Then, the ZCS control can be recovered after a short time, say $t_{S}$. This time can be selected around $T_{0}=2 \pi / \omega_{0}$ or longer. In the above example, $T_{0} \sim 2 \mu \mathrm{s}$, and the limit cycle is attained by using $t_{S}=2.5 \mu \mathrm{s}$. Actually, the steady oscillation is guaranteed if $a_{v}$ is reset to zero after the first switching is produced.

(b) A second example is shown in the two central panels of Fig. 9, also with the parameters given in Table 1, $R_{o s}=0$ and $R_{o p}=50 \Omega$, thus corresponding to a quality factor $Q_{2}=1.5624$, with associated critical values $\beta_{h c}=0.3165, \beta_{c c}=0.6331$ and $\beta_{s n}=0.6496$. Notice that in this case, the quality factor is too low to permit the ZCS control to be restored after the transient with voltage feedback. Hence, simulations have been performed by using $a_{v} / a_{i}=20 \mathrm{mV} / \mathrm{A}$ if $0<t<t_{S}$ (then $\beta \sim 0<\beta_{h c}\left(Q_{2}\right)$ ) and $a_{v} / a_{i}=10 \mathrm{mV} / \mathrm{A}$ if $t>t_{S}$ (then $\left.\beta=0.4931<\beta_{s n}\left(Q_{2}\right)\right)$.

(c) The third example, which is shown in the two panels on the right of Fig. 9, uses $R_{o p}=15 \Omega$ and the other parameters are equal to those in the previous examples. Now, $Q_{3}=0.4769<1 / 2$, and consequently $\beta$ must be negative in order to make the equilibrium points virtual, thus forcing an oscillatory dynamics. The relation $a_{v} / a_{i}=70 \mathrm{mV} / \mathrm{A}$ has been selected so that $\beta=-0.0501<0$.

\subsection{Control for a constant amplitude of the output voltage}

In a parallel configuration, the amplitude of the output voltage is proportional to the input voltage $V_{g}$, and also barely proportional to the quality factor $Q$ under ZCS control. Furthermore, the voltage feedback produces a variation in the amplitude of the voltage output, as it can be appreciated in Fig. 10(a), for parameters in Table $1, R_{o p}=250 \Omega$ and $a_{v} / a_{i} \in(0,0.5)$. It is then possible to get a benefit from this dependence in order to achieve a nearly constant output voltage if there are variations in the main voltage or even in the value of the load resistance. To achieve this goal, we can get a smooth signal by cyclically sampling the output voltage at its maximum value per cycle and filtering the sampled signal. Then, this filtered signal is subtracted from a reference equal to the desired output amplitude, and from this result multiplied by a proper factor $\chi, a_{v}$ is obtained. The idea is that $a_{v}$ is self-adjusted, such that the amplitude of $v_{o}$ (say $V_{o}$ ) is barely regulated to the chosen reference.

Diagrams in Fig. 10 (b-c) are obtained with a variable voltage input $V_{g} \in(10,80) \mathrm{V}$, a reference for the amplitude equal to $50 \mathrm{~V}$, and a feedback factor $\chi=0.07$. The relation $a_{v} / a_{i}$ in panel (b) is the value automatically established by the feedback. Diagram in panel (c) stands for the voltage output amplitude $V_{o}$. Notice the small variations in $V_{o}$ regarding the chosen reference in spite of the great variations of $V_{g}$. Actually, these variations can be further diminished by increasing the factor $\chi$, but then low frequency oscillations can take place due to instabilities. Furthermore, a similar feedback circuitry can be employed to stabilize the current output or the RMS value of the output voltage or the oscillation frequency. A more rigorous analysis of the inverter with amplitude or other variable regulation will be performed in a future work.

\section{Comparison with some frequency-domain-based methods}

Since the system studied in this paper can be considered as a linear system with relay feedback without hysteresis, and the above results are mathematically speaking completely rigorous, it turns interesting to compare them with the ones obtained from two frequency-domain-based methods of analysis of relay feedback systems. The first method is the widely used DF technique [13], also known as FHA in the context of resonant inverters. The second method is the exact frequency-domain technique for prediction of limit cycles in linear systems with relay feedback known as locus of perturbed relay system (LPRS) approach [16]. 


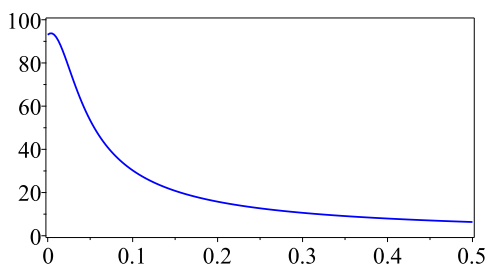

(a) $V_{o}$ versus $a_{v} / a_{i}$

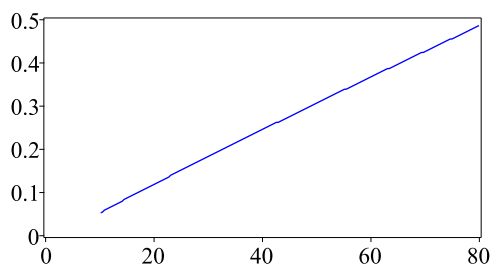

(b) $a_{v} / a_{i}$ versus $V_{g}$

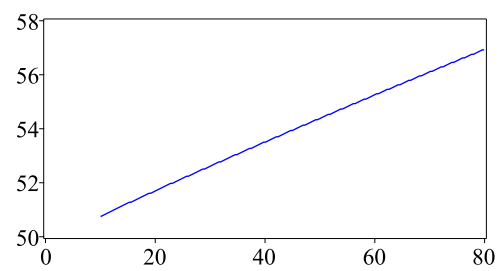

(c) $V_{o}$ versus $V_{g}$

Figure 10. Amplitude $V_{o}(\mathrm{~V})$ of the output voltage versus $a_{v} / a_{i}$ for the parallel configuration (a). Ratio $a_{v} / a_{i}$ (b) and $V_{o}$ (c) versus the input voltage $V_{g}$, with reference voltage equal to $50 \mathrm{~V}$ and factor $\chi=0.07$. Parameters are given in Table 1 and $R_{o p}=250 \Omega$.

\subsection{First harmonic approximation}

First, the approximate DF or FHA method is addressed and applied to system (7)-(8). We refer to [13] where it was shown that with this method, it is possible to detect in an approximate way several bifurcations involving limit cycles for third order relay feedback systems. It will be shown, however, that for the second order system considered in this study, the DF method is able to predict only a pale image of the actual nonlinear phenomena detected in this work.

System (7)-(8), which can be written in the following form

$$
\dot{\tilde{\mathbf{x}}}=\tilde{\mathbf{A}} \tilde{\mathbf{x}}+\operatorname{sign}\left(\mathbf{c}^{\top} \tilde{\mathbf{x}}\right) \tilde{\mathbf{b}},
$$

where $\mathbf{c}^{\top}=(0,1)$ and time $t$ corresponds to normalized one $\tau$, can be thought as a linear system with relay feedback, where the output $y=\mathbf{c}^{\top} \tilde{\mathbf{x}}=\tilde{x}_{2}$ is the feedback variable used to generate the input signal $u=\operatorname{sign}(y)$.

The DF method is based on the assumption that in the equivalent scalar differential equation for system (7)

$$
\left(D^{2}+\frac{1}{Q} D+1\right) y(t)+\left(-D-\frac{\beta}{Q}\right) u(t)=0
$$

where $D$ is the time differential operator, if we postulate a periodic solution in the form $y=A+B \cos (\omega t)$ with $B, \omega>0$, then the equation must be fulfilled when substituting $u(t)=\operatorname{sign}(y(t))$ by the first harmonic component of this last expression. In fact, if we define the bias and first harmonic gains of the relay as

$$
N_{0}(A, B)=\frac{1}{2 \pi A} \int_{-\pi}^{\pi} \operatorname{sign}(A+B \cos \theta) d \theta
$$

and

$$
N_{1}(A, B)=\frac{1}{\pi B} \int_{-\pi}^{\pi} \operatorname{sign}(A+B \cos \theta) \cos \theta d \theta
$$

then we can write

$$
u(t) \approx N_{0}(A, B) A+N_{1}(A, B) B \cos \omega t .
$$

Standard computations for $|A| \leq B$ give

$$
N_{0}(A, B)=\frac{2}{\pi A} \varphi, \quad N_{1}(A, B)=\frac{4}{\pi B} \cos \varphi,
$$

where

$$
\varphi=\arcsin \left(\frac{A}{B}\right) \in\left(-\frac{\pi}{2}, \frac{\pi}{2}\right)
$$

Otherwise, for $|A|>B$ one gets $N_{0}(A, B)=\operatorname{sign}(A) / A$ and $N_{1}(A, B)=0$.

Note now that from the scalar differential equation (25), it can be obtained the transfer function

$$
W(s)=-\frac{Y(s)}{U(s)}=\mathbf{c}^{\boldsymbol{\top}}(\tilde{\mathbf{A}}-s \mathbf{I})^{-1} \tilde{\mathbf{b}}=-\frac{s+\frac{\beta}{Q}}{s^{2}+\frac{1}{Q} s+1},
$$


where I stands for the identity matrix, so that the balance conditions can be reformulated in the form

$$
\begin{aligned}
A\left(1+W(0) N_{0}(A, B)\right) & =0, \\
1+W(j \omega) N_{1}(A, B) & =0,
\end{aligned}
$$

where $j=\sqrt{-1}$.

The conditions in (26)-(27) predict symmetric periodic solutions for $A=\varphi=0$ and $B=-4 W\left(j \omega^{*}\right) / \pi$, being $\omega^{*}>0$ the only solution of $\operatorname{Im} W(j \omega)=0$, which is equivalent to the condition

$$
\omega^{2}+\frac{\beta}{Q^{2}}=1
$$

This last condition implies that no symmetric periodic orbits are allowed for $\beta \geq Q^{2}$ but one symmetric periodic orbit should exist otherwise.

Moreover, if $A \neq 0$ is assumed, the balance conditions (26)-(27) lead to

$$
A=-\frac{2}{\pi} W(0) \varphi=\frac{2 \beta}{\pi Q} \varphi, \quad B=-\frac{4}{\pi} W\left(j \omega^{*}\right) \cos \varphi=\frac{4 Q}{\pi} \cos \varphi,
$$

where $\omega^{*}>0$, as before, satisfies $\operatorname{Im} W\left(j \omega^{*}\right)=0$. Dividing these expressions and rearranging terms, we get the condition for existence of non-symmetric periodic orbits

$$
\sin (2 \varphi)=\frac{\beta}{Q^{2}} \varphi
$$

which, as long as $0<\beta<Q^{2}$, predicts the existence of a symmetric pair of non-symmetric periodic orbits.

In short, the predictions based on the DF method imply one symmetric periodic orbit for $\beta<0$, three periodic orbits (one symmetric and two non-symmetric but symmetric each other) for $0<\beta<Q^{2}$, and none for $\beta>Q^{2}$. These predictions are rather poor, when compared with the real bifurcation lines in Fig. 5. In particular, the transition from the existence of the three periodic orbits to only two symmetric periodic orbits (that is the homoclinic connection) is not detected, and the bifurcation line leading to the parameter region with no periodic orbits is very roughly approximated.

\subsection{Locus of a perturbed relay system method}

A more powerful tool to deal with relay system with feedback is the frequency-domain technique based on LPRS analysis detailed in [15] and applied in [16] to a buck converter under sliding mode control with hysteresis feedback.

The LPRS method developed for systems with relay feedback can readily be applied to system (2)-(3) or any of their equivalent normalized ones. For convenience, let us use the instance (10)-(11), which can be compacted in the form

$$
\dot{\mathbf{x}}=\mathbf{A x}+\operatorname{sign}\left(\mathbf{c}^{\top} \mathbf{x}\right) \mathbf{b},
$$

where time $t$ now corresponds to normalized one $\theta$. With this method, the following frequency-dependent complexvalued function is defined

$$
J(\omega)=\mathcal{R}(\omega)+j \mathcal{I}(\omega),
$$

where $\omega$ is the frequency normalized in accordance to time $\theta$, and $\mathcal{R}(\omega)$ and $\mathcal{I}(\omega)$ are the real and the imaginary parts of $J(\omega)$ that can be expressed, see [16], as follows

$$
\begin{aligned}
& \mathcal{R}(\omega)=-\frac{1}{2} \mathbf{c}^{\top}\left(\mathbf{A}^{-1}+\frac{2 \pi}{\omega}\left(\mathbf{I}-e^{\frac{2 \pi}{\omega} \mathbf{A}}\right)^{-1} e^{\frac{\pi}{\omega} \mathbf{A}}\right) \mathbf{b}, \\
& \mathcal{I}(\omega)=\frac{\pi}{4} \mathbf{c}^{\top}\left(\mathbf{I}+e^{\frac{\pi}{\omega} \mathbf{A}}\right)^{-1}\left(\mathbf{I}-e^{\frac{\pi}{\omega} \mathbf{A}}\right) \mathbf{A}^{-1} \mathbf{b} .
\end{aligned}
$$

Typically, the existence of a limit cycle can be checked by plotting the graph of the complex function $J(\omega)$ in terms of the frequency $\omega$. In our case, the more general expressions given in [15] for a relay control with hysteresis 


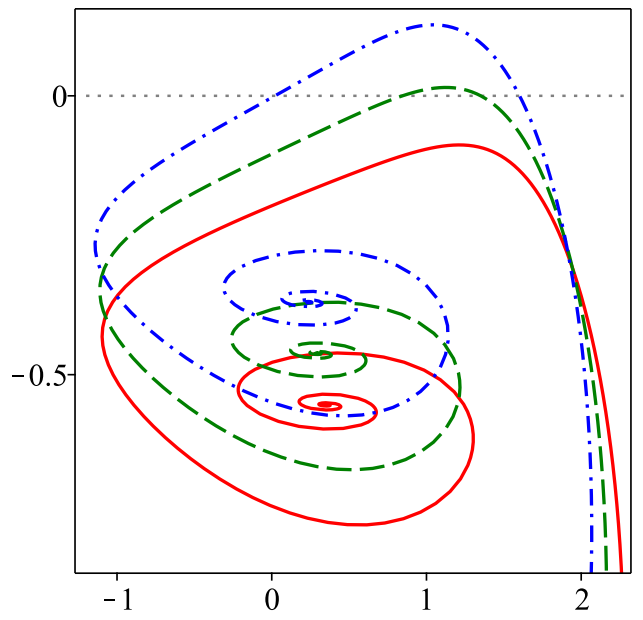

(a) The plot of $J(\omega)$ in the complex domain

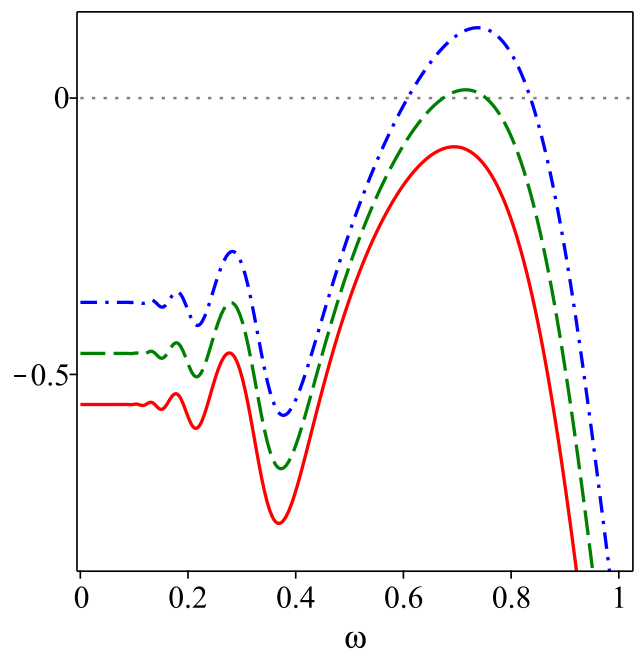

(b) The imaginary part $\mathcal{I}$ versus $\omega$

Figure 11. Diagrams for $J(\omega)$ in the complex domain in (a) and its imaginary part $\mathcal{I}(\omega)$ in (b). Parameters are $\gamma=-0.25$ and $\beta \in\{1.5,1.25,1.0\}$. Notice that continuous red curve for $\beta=1.5$ does not crosses the zero line for the imaginary part.

$b$ and amplitude $c$ can be applied here by considering $b=0$ and $c=1$. Therefore, according to reference [15], the oscillation frequency is determined at the point where the imaginary part $\mathcal{I}(\omega)$ is zero, that is to say when the plot of $J(\omega)$ in the complex domain crosses the real axis.

Note that (31) can be written in the form $\mathcal{I}(\omega)=\frac{\pi}{4} \mathbf{c}^{\top} \mathbf{X}$, in which $\mathbf{X}$ is the state vector at the crossing point of the limit cycle, i.e., lying in the switching line, whenever $\mathcal{I}(\omega)=0$. In our case, $\mathbf{c}^{\top}=(0,1)$ and then $\mathbf{X}=(z, 0)$, according to the notation introduced in Section 5.1. A question not clearly covered by the LPRS analysis concerns the admissibility of the limit cycles obtained, with such a general method, for particular values of matrices and vectors defining system (28). Noticing that the crossing limit cycle is a closed orbit with two symmetric parts lying each one on its corresponding region, a necessary condition for the crossing limit cycle to be admissible is that it crosses the switching line only at a pair of symmetric points, say $\mathbf{X}$ and $-\mathbf{X}$. Multiple possible mathematical solutions for $I(\omega)=0$ can coexist, but some of them could not be physically admissible, as it is shown in the following examples.

Let us consider the focus case $(Q>1 / 2)$ with a representative parameter value $\gamma=-0.25$, for which the critical values $\beta_{s n}=1.2852$ and $\beta_{c c}=1.2167$ follow from Proposition 3. In Fig. 11, $J(\omega)$ is represented in the complex plane in panel (a) and its imaginary part $\mathcal{I}(\omega)$ is shown in panel (b), for $\beta \in\{1.5,1.25,1.0\}$. Notice the different behavior for these three particular values of the parameter $\beta$. If $\beta=1.5>\beta_{s n}$, there is no solution for $\omega$ for the equation $\mathcal{I}(\omega)=0$, then crossing limit cycles cannot exist. In the other two cases, two mathematical solutions meet condition $\mathcal{I}(\omega)=0$, and the one with the highest value of $\omega$ is admissible and stands for the stable crossing limit cycle. However, the other solution with a lower frequency $\omega$ is valid for $\beta_{s n}>\beta=1.25>\beta_{c c}$, but not for $\beta=1.0<\beta_{c c}$, in accordance to Proposition 3. In Fig. 12, the corresponding cycles are shown with $\beta=1.25$ in panel (a) and $\beta=1.0$ in panel (b). To get these cycles, we have used the particular solution for vector $\mathbf{X}$ at frequencies satisfying $\mathcal{I}(\omega)=0$. It can be appreciated the multiple crossing at the switching line of the inner cycle in panel (b), so it is not admissible. In this case, the unstable limit cycle is of sliding type, as it can be deduced and obtained from Proposition 7. However, from the LPRS method, sliding limit cycles cannot be concluded. ${ }^{4}$

Let illustrate now a case with an extra pair of solutions for condition $\mathcal{I}(\omega)=0$, by using also $\gamma=-0.25$ and a lower value $\beta=0.20$ for the other parameter, as it can be observed in the graph of $\mathcal{I}(\omega)$ given in Fig. 13(a). This

\footnotetext{
${ }^{4}$ The authors are aware that the LPRS method is able to predict attracting limit cycles of sliding type when a hysteresis decision is used in the feedback. Note that, for the kind of the considered systems in this study, namely, resonant inverters, the switching law is selected in such a way that the resulting desired orbits are of crossing (not sliding) type and that with this control law, notice that the minus operator is omitted for $u=\operatorname{sign}\left(\mathrm{x}_{2}\right)$ in (10)-(11), the sliding trajectories on the switching boundary are repulsive.
} 


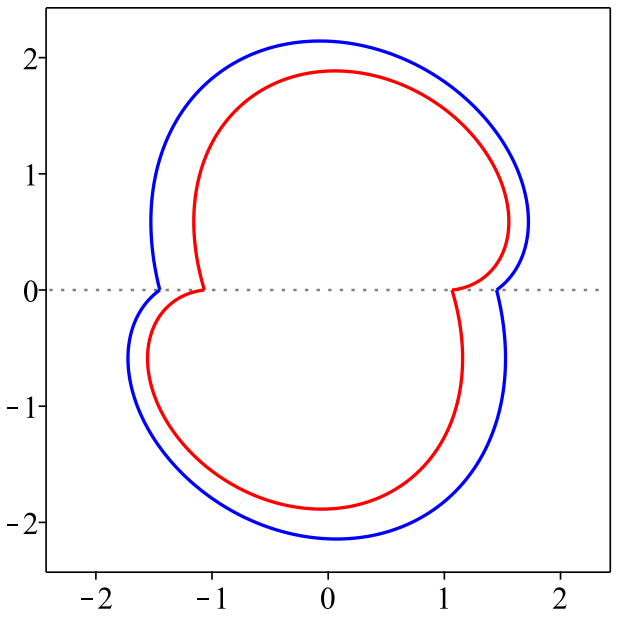

(a) $\beta=1.25, \omega=\{0.7534,0.6738\}$

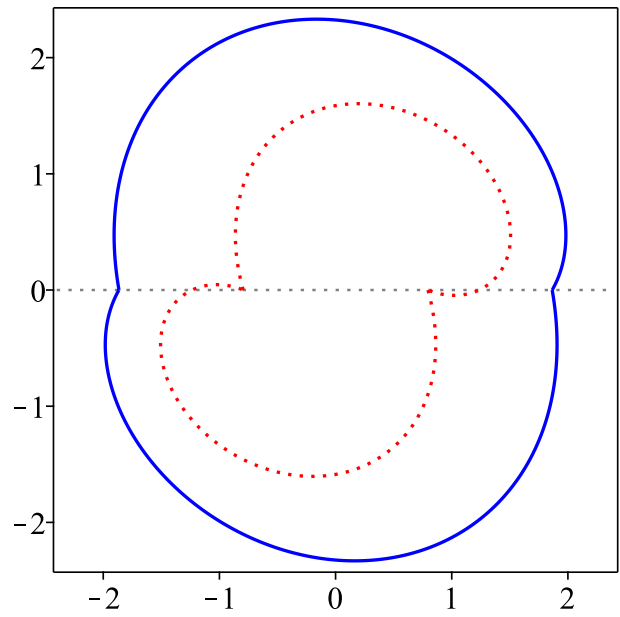

(b) $\beta=1.0, \omega=\{0.8360,0.6078\}$

Figure 12. Limit cycles using parameter $\gamma=-0.25$, with parameter $\beta$ and normalized frequency values given in the caption. The blue continuous lines stand for real stable crossing limit cycles, the red continuous line in (a) is the unstable crossing limit cycle and the red dotted line in (b) is a 'ghost' or not valid cycle, due to having more than two points in common with the switching line.

function crosses through the zero line at four frequency instances, which associated cycles are represented in panels (b-c) in the same figure. Note that only the cycle corresponding to the higher frequency crossing, at $\omega=0.9732$, is admissible. The remainder cycles with lower frequency have more than one pair of crossing points with the switching line, thus being non-admissible.

Finally, it is interesting to notice that LPRS method can be used to compute the saddle-node bifurcation, at which the stable and unstable crossing limit cycles collapse, by solving the system of two nonlinear equations $\mathcal{I}(\omega)=0$ and $d I(\omega) / d \omega=0$, so that $I(\omega)$ collides tangentially to the zero line. The result obtained, by using $\gamma=-0.25$, is the pair of values $\beta=1.2852=\beta_{s n}$ and $\omega=0.7124=\pi / \theta_{M}$, where $\theta_{M}=4.4100$ can be calculated from (21).

Summarizing, the LPRS method gives exact values of frequencies for crossing limit cycles from condition $\mathcal{I}(\omega)=$ 0 , although the validity of the associated cycles must be tested by plotting them in order to check that they cross the switching line only at a pair of symmetric points. Moreover, the saddle-node bifurcation of cycles can be exactly computed in the frame of LPRS method in a clear contrast to the DF method, which only gives an estimate of where this bifurcation could take place. However, by its nature, the LPRS method does not account for sliding limit cycles, which in our case are unstable and related to a rejecting sliding segment, thus playing a relevant role in defining the basin of multiple attractors of the system.

\section{Conclusions}

A self-oscillating H-bridge LC resonant inverter with state feedback control has been analyzed in this paper. The full physical parameter set, thus including parameters in the two plant and control stages, has been reduced to only two parameters. These are the quality factor $Q$ of the load and LC resonator, and the parameter $\beta$, which accounts for the parallel or series character of the inverter and for the control gains. In this reduced parameter plane, five bifurcation lines, which emerge from the singular point $(Q, \beta)=(1 / 2,0)$, have been found, thus defining an equivalent number of parameter regions, each one showing specific dynamical features. The obtained expressions for these critical lines can help the designers to properly select the physical parameters in order to ensure the desired oscillatory behavior. Moreover, the use of state feedback allows the oscillation under any value of the quality factor, even in the case of over-damping $(Q<1 / 2)$. We emphasize the fact that the mentioned critical lines are defined for conditions involving either the equilibrium, the crossing or the sliding limit cycles. Consequently, they affect not only the stable crossing limit cycle existence, but also they determine the reachability of this cycle depending on the initial conditions. In 


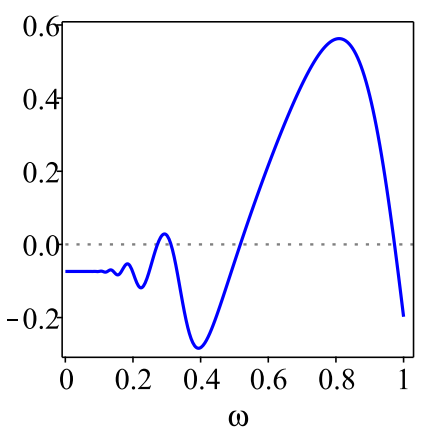

(a) $I(\omega)$

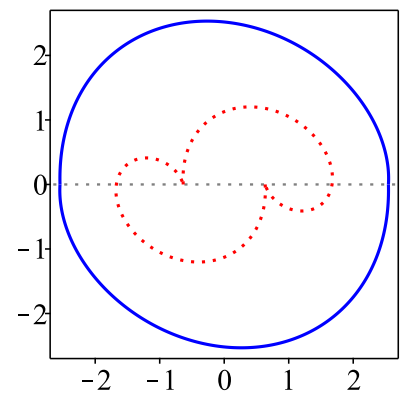

(b) $\omega=\{0.9732,0.5174\}$

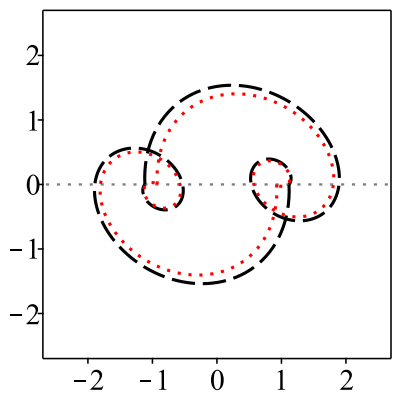

(c) $\omega=\{0.3123,0.2724\}$

Figure 13. Plot of $\mathcal{I}(\omega)$ in (a), using parameters $\gamma=-0.25$ and $\beta=0.20$. Limit cycles for normalized frequencies in the caption such that $\mathcal{I}(\omega)=0$, are represented in (b-c). Note that only the biggest crossing limit cycle in (b), for $\omega=0.9732$, is admissible. The remainder cycles have more than two crossing points with the switching line, thus being invalid.

this sense, some ideas, involving the control parameters, are suggested in the last part of the paper in order to ensure oscillation for low values of the quality factor. Finally, from the comparison of the analysis exposed in this paper, based on time-domain differential equations in the state-plane, it is shown that DF and LPRS frequency-domain techniques fail in predicting sliding limit cycles and their bifurcations, what emphasizes the relevance of the dynamical systems approach developed in this work.

\section{Acknowledgments}

This work has been sponsored by the Spanish Agencia Estatal de Investigación (AEI) and the Fondo Europeo de Desarrollo Regional (FEDER) under grants DPI2017-84572-C2-1-R and DPI2016-80491-R (AEI/FEDER, UE) and also by the Spanish Ministerio de Economía y Competitividad, in the frame of project MTM2015-65608-P and by the Consejería de Economía y Conocimiento de la Junta de Andalucía under grant P12-FQM-1658.

\section{References}

[1] R. W. Erickson, D. Maksimovic, Fundamentals of power electronics, Lluwer, Springer, 2001.

[2] R. Oruganti, F. C. Lee, Resonant power processors, Part I-State plane analysis, IEEE Trans. on Ind. Appl. 6 (1985) 1453-1460.

[3] V. Volpérian, Approximate small-signal analysis of the series and the parallel resonant converters, IEEE Trans. on Power Electron. 4 (1) (1989) $15-24$

[4] S. Y. Yu, R. Chen, A. Viswanathan, Survey of Resonant Converter Topologies, 2018 Texas Instruments, Power Supply Design Seminar SEM2300, TI Literature Number SLUP376, https://www.ti.com/seclit/ml/slup376/slup376.pdf.

[5] T. Qian, A converter combination scheme for efficiency improvement of PV systems, IEEE Trans. Circuits Syst. II, Exp. Briefs 65 (11) (2018) 1668-1672.

[6] C. H. Chang, E. C. Chang, C. A Cheng, H. L. Cheng, S. C. Lin, Small Signal Modeling of LLC Resonant Converters Based on Extended Describing Function, Int. Symp, on Computer, Consumer and Control (2012) 365-368.

[7] T. S. Chan, C. L. Chen, A primary side control method for wireless energy transmission system, IEEE Trans. Circ. Syst. I 59 (8) (2012) 1805-1814.

[8] R. Yang, H. Ding, Y. Xu, L. Yao, Y. Xiang, An analytical steady-state model of LCC type series-parallel resonant converter with capacitive output filter, IEEE Trans. Power Electron. 29 (1) (2014) 328-338.

[9] R. Bonache-Samaniego, C. Olalla, L. Martínez-Salamero, Design of self-oscillating resonant converters based on a variable structure systems approach, IET Power Electron. 57, (1) (2015) 111-119.

[10] R. Bonache-Samaniego, C. Olalla, L. Martínez-Salamero, Dynamic Modeling and Control of Self-Oscillating Parallel Resonant Converters Based on a Variable Structure Systems Approach, IEEE Trans. on Power Electron. 32 (2) (2017) 1469-1480.

[11] D. Williams, C. Bingham, M. Foster, D. Stone, Hamel locus design of self-oscillating DC-DC resonant converters, IET Power Electron. 3 (1) (2010) 86-94.

[12] H. Pinheiro, P. K. Jain, G. Joos, Self-sustained oscillating resonant converters operating above the resonant frequency, IEEE Trans. on Power Electron. 14 (5) (1999) 803-815.

[13] R. Genesio, G. Bagni, A View on Limit Cycle Bifurcations in Relay Feedback Systems, IEEE Trans. Circ. Syst. I 50 (8) (2003) $1134-1140$.

[14] J. Mućko, R. Strzelecki, Errors in the analysis of series resonant inverter/converter assuming sinusoidal waveforms of voltage and current, 10th Int. Conf. on Compatibility, Power Electron. and Power Eng. (CPE-POWERENG). DOI: 10.1109/CPE.2016.7544216. 
[15] I. Boiko, Oscillations and transfer properties of relay servo systems - the locus of a perturbed relay system approach. Automatica, 41 (4) (2005) 677-683

[16] I Boiko, LPRS analysis of sliding mode buck converter, Journal of the Franklin Inst. 353 (18) (2016) 5137-5150

[17] M. G. Kim, D. S. Lee, M. J. Youn, A New State Feedback Control of Resonant Converters, IEEE Trans. on Ind. Electron. 38 (3) (1991) $173-179$.

[18] C. S. Tang, Y. Sun, Y. G. Su, S. K. Nguang, A. P. Hu, Determining multiple steady-state ZCS operating points of a switch-mode contactless power transfer system, IEEE Trans. on Power Electron. 24 (2) (2009) 416-425.

[19] V. I. Utkin, Variable structure systems with sliding modes, IEEE Trans. on Automatic Control 22 (2) (1997) $212-222$.

[20] V. I. Utkin, Sliding mode control of DC/DC converters, Journal of the Franklin Inst. 350 (8) (2013) $2146-2165$.

[21] E. Freire, E. Ponce, F. Torres, A general mechanism to generate three limit cycles in planar Filippov systems with two zones. Nonlinear Dynamics 78 (2014) 251-263.

[22] L. Benadero, E. Ponce, A. El Aroudi, F. Torres, Limit Cycle Bifurcations in Resonant LC Power Inverters under Zero Current Switching Strategy, Nonlinear Dynamics 91 (2) (2018) 1145-1161.

[23] A. El Aroudi, L. Benadero, E. Ponce, C. Olalla, F. Torres, L. Martinez-Salamero, Nonlinear Dynamic Modeling and Analysis of SelfOscillating H-Bridge Parallel Resonant Converter Under Zero Current Switching Control: Unveiling Coexistence of Attractors, IEEE Transactions on Circuits and Systems I: Regular Papers 66 (4) (2019) 1657-1667.

[24] A. El Aroudi, L. Benadero, E. Ponce, C. Olalla, F. Torres, L. Martinez-Salamero, Suppression of Undesired Attractors in a Self-Oscillating H-Bridge Parallel Resonant Converters Under Zero Current Switching Control, IEEE Trans. Circuits Syst. II, Exp. Briefs 66 (4) (2019) 692696.

[25] O. Lucia, J. M. Burdio, I. Millan, J. Acero, D. Puyal, Load-Adaptive Control Algorithm of Half-Bridge Series Resonant Inverter for Domestic Induction Heating, IEEE Transactions on Industrial Electronics 56 (8) (2009) 3106--3116.

[26] A. A. Andronov, A. Vitt, S. E. Khaikin, Theory of oscillators, Pergamon Press, Oxford, 1966.

[27] Yu. A. Kuznetsov, S. Rinaldi, A. Gragnani, One-Parameter Bifurcations in Planar Filippov Systems, Int. J. Bifurc. and Chaos 13 (2003) 2157-2188.

[28] E. Freire, E. Ponce, F. Torres, On the critical crossing cycle bifurcation in planar Filippov systems, J. Differ. Equations 259 (2015) $7086-7107$. 